\title{
A SHARP REMEZ INEQUALITY FOR TRIGONOMETRIC POLYNOMIALS
}

\author{
E. NURSULTANOV AND S. TIKHONOV
}

ABStRACt. We obtain a sharp Remez inequality for the trigonometric polynomial $T_{n}$ of degree $n$ on $[0,2 \pi)$ :

$$
\left\|T_{n}\right\|_{L_{\infty}([0,2 \pi))} \leqslant\left(1+2 \tan ^{2} \frac{n \beta}{4 m}\right)\left\|T_{n}\right\|_{L_{\infty}([0,2 \pi) \backslash B)},
$$

where $\frac{2 \pi}{m}$ is the minimal period of $T_{n}$ and $|B|=\beta<\frac{2 \pi m}{n}$ is a measurable subset of $\mathbb{T}$. In particular, this gives the asymptotics of the sharp constant in the Remez inequality:

$$
\mathcal{C}(n, \beta)=1+\frac{(n \beta)^{2}}{8}+O(\beta)^{4},
$$

where

$$
\mathcal{C}(n, \beta):=\sup _{|B|=\beta} \sup _{T_{n}} \frac{\left\|T_{n}\right\|_{L_{\infty}([0,2 \pi))}}{\left\|T_{n}\right\|_{L_{\infty}([0,2 \pi) \backslash B)}} .
$$

We also obtain sharp Nikol'skii's inequalities for the Lorentz spaces and Net spaces. Multidimensional variants of Remez and Nikol'skii's inequalities are investigated.

\section{INTRODUCTION}

Consider the space of (complex) trigonometric polynomials of degree at most $n \in \mathbb{N}$, i.e.,

$$
\mathfrak{N}_{n}=\left\{T_{n}: \quad T_{n}(x)=\sum_{|k| \leqslant n} c_{k} e^{i k x}, \quad c_{k} \in \mathbb{C}, \quad x \in \mathbb{T}\right\} .
$$

An important question in polynomial approximation is the following one. Let $|B|=\mu(B)$ be the linear Lebesgue measure of $B$. How large can $\left\|T_{n}\right\|_{L_{\infty}([0,2 \pi))}$ be if

$$
\left|\left\{x \in[0,2 \pi):\left|T_{n}(x)\right|>1\right\}\right| \leqslant \varepsilon
$$

holds for some $0<\varepsilon \leqslant 1$ ? The well-known Remez inequality answers this question.

For any Lebesgue measurable set $B \subset \mathbb{T}$ such that $|B| \equiv \beta<\pi / 2$ we have

$$
\left\|T_{n}\right\|_{L_{\infty}([0,2 \pi))} \leqslant C(n,|B|)\left\|T_{n}\right\|_{L_{\infty}([0,2 \pi) \backslash B)}, \quad T_{n} \in \mathfrak{N}_{n}
$$

We will write $\mathcal{C}(n,|B|)$ in place of $C(n,|B|)$ while dealing with the sharp constant in this estimate, see (0.1).

Investigation of Remez's inequalities is a well developed topic. For algebraic polynomials sharp inequality was proved by Remez [Re]. In [BE] and [Er1] (1.2) was proved with $C(n,|B|)=\exp (4 n|B|)$; the history of the question can be found in e.g. [Er3, Sec. 2], [Ga, Sec. 3], and [LGM]. In [Ga, Th. 3.1], the constant was sharpened as $C(n,|B|)=\exp (2 n|B|)$. Also, another improvement was obtained in [Er2, Th. 3.4]: $C(n,|B|)=\exp \left(n\left(|B|+1.75|B|^{2}\right)\right)$.

In case of $B \equiv[a, b] \subset[-\pi, \pi]$ a sharp Remez inequality is given by (see [Er1])

$$
\left\|T_{n}\right\|_{L_{\infty}([0,2 \pi))} \leqslant \frac{1}{2}\left(\tan ^{2 n}\left(\frac{2 \pi-\beta}{8}\right)+\cot ^{2 n}\left(\frac{2 \pi-\beta}{8}\right)\right)\left\|T_{n}\right\|_{L_{\infty}([0,2 \pi) \backslash B)}, \quad|B|=\beta, \quad T_{n} \in \mathfrak{N}_{n}
$$

and equality in (1.3) holds if and only if

$$
T_{n}(x)=C \mathfrak{T}_{n}\left(\frac{\cos (x-(a+b) / 2)-\cos ^{2}((b-a) / 4)}{\sin ^{2}((b-a) / 4)}\right), \quad C \in \mathbb{R},
$$

Date: July 6, 2011.

2000 Mathematics Subject Classification. Primary 41A17, 41A44; Secondary 46E30.

Key words and phrases. Remez and Nikol'skii's inequalities, Trigonometric polynomials, Lorentz and Net spaces.

This research was partially supported by MTM 2011-27637, 2009 SGR 1303, RFFI 09-01-00175, and NSH-3252.2010.1. 
where $\mathfrak{T}_{n}(x)$ is the Chebyshev polynomial of degree $n$, i.e.,

$$
\mathfrak{T}_{n}(x)=\frac{1}{2}\left(\left(x+\sqrt{x^{2}-1}\right)^{n}+\left(x-\sqrt{x^{2}-1}\right)^{n}\right)
$$

for every $x \in \mathbb{R} \backslash(-1,1)$.

Therefore, the best known bounds of the sharp constant in the Remez inequality are given by

$$
\frac{1}{2}\left(\tan ^{2 n}\left(\frac{2 \pi-\beta}{8}\right)+\cot ^{2 n}\left(\frac{2 \pi-\beta}{8}\right)\right) \leqslant \mathcal{C}(n, \beta) \leqslant \exp (n \beta \min \{2,(1+1.75 \beta)\}),
$$

for $0<\beta \leqslant \pi / 2$. Moreover, Erdélyi [Er2] and Ganzburg [Ga] conjectured that

$$
\mathcal{C}(n, \beta)=\frac{1}{2}\left(\tan ^{2 n}\left(\frac{2 \pi-\beta}{8}\right)+\cot ^{2 n}\left(\frac{2 \pi-\beta}{8}\right)\right)
$$

for any $\beta>0$.

In case when the measure $|B|=\beta$ is big, that is, when $\pi / 2<\beta<2 \pi$, it is known that

$$
\mathcal{C}(n, \beta) \leqslant C(n, \beta)=\left(\frac{17}{2 \pi-\beta}\right)^{2 n}
$$

(see [Er1, Er2, Ga, Na]). Another upper bound of $\mathcal{C}(n, \beta), \beta>0$, was given by Andrievskii [An].

In this paper we investigate Remez's inequalities for the case of $0<\beta<\frac{2 \pi}{n}$. Note that in applications in fact we are usually interested in the case of small $\beta$ (see, e.g., $[\mathrm{MT}]$ ). Precisely, we prove

$$
\left\|T_{n}\right\|_{L_{\infty}([0,2 \pi))} \leqslant\left(1+2 \tan ^{2} \frac{n \beta}{4 m}\right)\left\|T_{n}\right\|_{L_{\infty}([0,2 \pi) \backslash B)}, \quad 0<\beta<\frac{2 \pi m}{n},
$$

where $\frac{2 \pi}{m}$ is the minimal period of $T_{n} \in \mathfrak{N}_{n}$. Equality in (1.6) holds for $T_{n}(x)=\cos n x+\frac{1}{2}\left(1-\cos \frac{\beta}{2}\right)$. This improves the right-hand side bound of the sharp constant in the Remes inequality:

$$
\mathcal{C}(n, \beta) \leqslant 1+2 \tan ^{2} \frac{n \beta}{4}, \quad 0<\beta<\frac{\pi}{n} .
$$

Combining the left-hand side of (1.4) and (1.7) we show (see Corollary 3.5) that

$$
\mathcal{C}(n, \beta)=1+\frac{(n \beta)^{2}}{8}+O\left(\beta^{4}\right) .
$$

This answers Stechkin-Ulyanov's question $([\mathrm{SU}])$ on the rate of decrease of $\mathcal{C}(n, \beta)$. Moreover,

which proves conjecture (1.5) for $n=1$.

$$
\mathcal{C}(1, \beta)=1+2 \tan ^{2} \frac{\beta}{4},
$$

The paper is organized as follows. In section 2, we prove several basic auxiliary results for rearrangements. We recall that the distribution of a measurable function $f$ on $\mathbb{T}$ is defined by $\lambda(\sigma, f)=\mu\{x \in[0,2 \pi]$ : $|f(x)|>\sigma\}$. Then $f^{*}(t)=\inf \{\sigma: \lambda(\sigma, f) \leqslant t\}$ is the decreasing rearrangement of $f$. We will also consider the decreasing rearrangement of functions on their minimal periods denoting it by $f^{\star}$.

In section 3, we prove sharp Remez and Remez-Bernstein's inequalities in terms of rearrangements and averages which are defined as follows. Any fixed family $M$ of measurable subsets of $\mathbb{T}$ is called a net in $\mathbb{T}$.

For a $2 \pi$-periodic locally integrable function $f$ we define the function

$$
\overline{f(t, M)}=\sup _{\substack{e \in M \\|e| \geqslant t}} \frac{1}{|e|}\left|\int_{e} f(x) d x\right|, \quad t \geqslant 0,
$$

which is called the average function of $f$ on the net $M$ ([NT]). If $\sup _{e \in M}|e|=\alpha$ and $t>\alpha$, then $\overline{f(t, M)}=0$.

In section 4, we obtain sharp Bernstein and Bernstein-Nikol'skii's inequalities in the Lorentz spaces, in particular Lebesgue spaces, and in the Net spaces. As usual, a measurable function $f$ defined on $\mathbb{T}$ belongs to the Lorentz space $L_{p, q}=L_{p, q}(\mathbb{T})$ (see e.g. [BS]) if

and

$$
\|f\|_{L_{p, q}}=\left(\int_{0}^{2 \pi}\left(t^{1 / p} f^{*}(t)\right)^{q} \frac{d t}{t}\right)^{1 / q}<\infty, \quad 0<p, q<\infty,
$$

$$
\|f\|_{L_{p, \infty}}=\sup _{t \in(0,2 \pi)} t^{1 / p} f^{*}(t)<\infty, \quad 0<p \leqslant \infty .
$$


The scale of net spaces is more general than the scale of Lorentz spaces. To define them, let $0<p, q \leqslant \infty$, $M$ be a net in $\mathbb{T}$, and a $2 \pi$-periodic function $f$ be locally integrable. We say that $f$ belongs to the Net space $N_{p, q}(M)=N_{p, q}(M, \mathbb{T})([\mathrm{NA}, \mathrm{NT}])$ if

$$
\|f\|_{N_{p, q}(M)}=\left(\int_{0}^{2 \pi}\left(t^{1 / p} \overline{f(t, M)}\right)^{q} \frac{d t}{t}\right)^{1 / q}<\infty, \quad 0<p, q<\infty
$$

and

$$
\|f\|_{N_{p, \infty}(M)}=\sup _{t \in(0,2 \pi)} t^{1 / p} \overline{f(t, M)}<\infty, \quad 0<p \leqslant \infty .
$$

The net spaces $N_{p, q}(M)$ are a natural generalization of the Lorentz spaces $L_{p, q}$, since if $M$ is a collection of all compact sets in $\mathbb{T}$, then $N_{p, q}(M)=L_{p, q}(\mathbb{T})$ for $1<p<\infty, 0<q \leqslant \infty$.

Finally, multidimensional variants of Remez and Nikol'skii's inequalities are obtained in Section 5 . In particular, for trigonometric polynomials

$$
T_{\mathbf{n}}(x)=\sum_{\left|k_{1}\right| \leqslant n_{1}} \ldots \sum_{\left|k_{d}\right| \leqslant n_{d}} c_{\mathbf{k}} e^{i(\mathbf{k}, x)}, \quad c_{\mathbf{k}} \in \mathbb{C}, \quad x \in \mathbb{T}^{d}, \quad d \geqslant 1,
$$

we obtain the following Remez inequality

$$
\left\|T_{\mathbf{n}}\right\|_{L_{\infty}\left(\mathbb{T}^{d}\right)} \leqslant \frac{1}{\cos \sqrt[d]{|B| \prod_{j=1}^{d} \frac{j n_{j}}{2}}}\left\|T_{\mathbf{n}}\right\|_{L_{\infty}\left(\mathbb{T}^{d} \backslash B\right)}, \quad 0 \leqslant|B|<\frac{\pi^{d}}{\prod_{j=1}^{d} j n_{j}}
$$

cf. $[\mathrm{DP}]$ and $[\mathrm{Kr}]$.

\section{BASIC LEMMAS ON REARRANGEMENTS}

Let $T_{n} \in \mathfrak{N}_{n}$ and $d=d\left(T_{n}\right)$ be the minimal period of $T_{n}$. Note that $\frac{2 \pi}{n} \leqslant d \leqslant 2 \pi$. Let $T_{n}^{\star}(t)$ be the decreasing rearrangement of $T_{n}$ on its minimal period, i.e.,

$$
\lambda_{\min }\left(\sigma, T_{n}\right)=\left|\left\{x \in[0, d):\left|T_{n}(x)\right|>\sigma\right\}\right|
$$

and

$$
T_{n}^{\star}(t)=\inf \left\{\sigma: \lambda_{\min }\left(\sigma, T_{n}\right) \leqslant t\right\} .
$$

Remark that this concept describes the order of polynomials more accurately than $T_{n}^{*}$. For example,

$$
(\cos n x)^{*}(\beta)=(\cos x)^{*}(\beta)=\cos \frac{\beta}{4}, \quad 0 \leqslant \beta<2 \pi
$$

but

$$
(\cos n x)^{\star}(\beta)=\cos \frac{n \beta}{4}, \quad 0 \leqslant \beta<\frac{2 \pi}{n} .
$$

Moreover, the following result holds.

Lemma 2.1. Let $n \in \mathbb{N}$ and $f \in \mathfrak{N}_{n}$ be such that $\left|c_{n}(f)\right|+\left|c_{-n}(f)\right|>0$. If $d$ is the minimal period of $f$, then

(a) we have $f^{\star}(\beta) \leqslant f^{*}(\beta)$ for $0<\beta \leqslant d$ and $f^{\star}(\beta)<f^{*}(\beta)$ for $d<2 \pi$;

(b) there exists $m \in \mathbb{N}$ such that $d=2 \pi / m$;

(c) we have $n / m \in \mathbb{N}$ and $g(x)=f(x / m)$ is a trigonometric polynomial of degree $n / m$;

(d) $f^{*}(\beta)=g^{*}(\beta), \beta \in[0,2 \pi)$;

(e) $f^{\star}(\beta)=f^{*}(m \beta)$ for $0 \leqslant \beta \leqslant d$.

Proof. Item (a) follows from $\lambda_{\min }(\sigma, f) \leqslant \lambda(\sigma, f)$ and $\lambda_{\min }(\sigma, f)<\lambda(\sigma, f)$ for $d<2 \pi$.

(b). If $d \neq 2 \pi / m, m \in \mathbb{N}$, then we take an integer $r$ such that $r d \leqslant 2 \pi<(r+1) d$. Suppose that $\alpha=2 \pi-r d$; then $f(x)=f(x+r d), x \in \mathbb{R}$. Using $2 \pi$-periodicity, we get $f(x)=f(x+2 \pi)=f(x+\alpha+r d)$, $x \in \mathbb{R}$. Then $f(x)=f(x+\alpha)$, which contradicts $\alpha<d$. 
(c) Let $d=2 \pi / m$ be the minimal period of $f$. Putting $g(x)=f(x / m), x \in[0,2 \pi)$, we have

$$
\begin{aligned}
c_{r}(f)=\int_{0}^{2 \pi} f(x) e^{-i r x} d x & =\sum_{k=0}^{m-1} \int_{2 \pi k / m}^{2 \pi(k+1) / m} f(x) e^{-i r x} d x=\sum_{k=0}^{m-1} \int_{0}^{2 \pi / m} f(x) e^{-i r(x+2 \pi k / m)} d x \\
& =\int_{0}^{2 \pi / m} f(x) e^{-i r x} d x\left(\sum_{k=0}^{m-1} e^{-2 \pi i k r / m)}\right) .
\end{aligned}
$$

Taking into account $\sum_{k=0}^{m-1} e^{-2 \pi i k r / m}=\left\{\begin{array}{ll}m, & \text { for } r=m s, s \in \mathbb{N}, \\ 0, & \text { otherwise }\end{array}\right.$, we get $c_{r}(f)=0$ for $r \neq m s$. Since $\left|c_{n}(f)\right|+\left|c_{-n}(f)\right|>0$, then $n / m \in \mathbb{N}$. Therefore, using (2.2) we get

$$
c_{m s}(f)=m \int_{0}^{2 \pi / m} f(x) e^{-i m s x} d x=\int_{0}^{2 \pi} f(x / m) e^{-i s x} d x=c_{s}(g),
$$

and $g$ is the trigonometric polynomial of degree $n / m$.

(d) Making use of $2 \pi / m$-periodicity, we get

$$
\begin{aligned}
\lambda(\alpha, f) & =|\{x \in[0,2 \pi]:|f(x)|>\alpha\}|=m|\{x \in[0,2 \pi / m]:|f(x)|>\alpha\}| \\
& =|\{x \in[0,2 \pi]:|f(x / m)|>\alpha\}|=|\{x \in[0,2 \pi]:|g(x)|>\alpha\}|=\lambda(\alpha, g) .
\end{aligned}
$$

Then $g^{*}(\beta)=f^{*}(\beta)$ for any $\beta \in[0,2 \pi]$.

(e) We proved that $d=\frac{2 \pi}{m}$ and $\lambda_{\min }(\sigma, f)=\frac{1}{m} \lambda(\sigma, f)$. Hence, for $0<\beta<d=\frac{2 \pi}{m}$,

$$
f^{\star}(\beta)=\inf \left\{\sigma: \lambda_{\min }(\sigma, f) \leqslant \beta\right\}=\inf \{\sigma: \lambda(\sigma, f) \leqslant m \beta\}=f^{*}(m \beta) .
$$

We denote by $\mathfrak{M}_{n}$ the set of real trigonometric polynomials, i.e.,

$$
\mathfrak{M}_{n}=\left\{T_{n} \in \mathfrak{N}_{n}: \quad T_{n}(x) \in \mathbb{R}, \quad x \in \mathbb{T}\right\} .
$$

Let us prove two auxiliary results on rearrangements. First, we recall the following known lemma. The proof can be found in the papers [Be] by S. Bernstein and [St] by S. Stechkin.

Lemma 2.2. For $T_{n} \in \mathfrak{M}_{n}$ such that $\left|T_{n}(x)\right| \leqslant 1$ and $T_{n}(0)=1$, we have

$$
T_{n}(x) \geqslant \cos n x, \quad-\frac{\pi}{n} \leqslant x \leqslant \frac{\pi}{n} .
$$

Lemma 2.3. Let $T_{n} \in \mathfrak{M}_{n}$ be such that

$$
\max T_{n}(x)-\min T_{n}(x)=2 .
$$

Then for $0 \leqslant \beta<\frac{2 \pi}{n}$,

$$
T_{n}^{\star}(\beta) \geqslant(\cos n x+\alpha)^{\star}(\beta),
$$

where $\alpha=\max T_{n}(x)-1$.

Proof. Denote

$$
\Xi:=\max T_{n}(x) \quad \text { and } \quad \xi:=\min T_{n}(x) .
$$

Let $d$ be the minimal period of $T_{n}$. Then there exist $x_{1}, x_{2} \in[0, d)$ such that $T_{n}\left(x_{1}\right)=\Xi, T_{n}\left(x_{2}\right)=\xi$ and the polynomial

$$
\widetilde{T}_{n}(x)=T_{n}\left(x_{1}-x\right)-\Xi+1
$$

satisfies all conditions of Lemma 2.2. Hence, for $0 \leqslant|x| \leqslant \frac{\pi}{n}$, it follows that

$$
\widetilde{T}_{n}(x) \geqslant \cos n x \text {. }
$$

Moreover, $\left|x_{1}-x_{2}\right| \geqslant \frac{\pi}{n}$ since if $\left|x_{1}-x_{2}\right|<\frac{\pi}{n}$, then

$$
\widetilde{T}_{n}\left(x_{1}-x_{2}\right)=T_{n}\left(x_{2}\right)-\Xi+1=-1<\cos n x,
$$

which contradicts (2.6). Inequality (2.6) can be written as follows

$$
T_{n}\left(x_{1}-x\right) \geqslant \cos n x+\alpha,
$$

where $\alpha=\Xi-1=\xi+1$. 
Similarly, Lemma 2.2 gives

$$
T_{n}\left(x_{2}-x\right) \leqslant \cos n\left(\frac{\pi}{n}-x\right)+\alpha=-\cos n x+\alpha, \quad 0 \leqslant|x| \leqslant \frac{\pi}{n}
$$

(here it is enough to consider $-T_{n}$ ).

Assume first that $\alpha \in[-1,1]$ and $0 \leqslant|x| \leqslant \frac{\arccos (-\alpha)}{n}$. Then using (2.7) we get

$$
T_{n}\left(x_{1}-x\right) \geqslant \cos n x+\alpha \geqslant 0 .
$$

Also using (2.8) for $\frac{\arccos (-\alpha)}{n} \leqslant x \leqslant \frac{2 \pi}{n}-\frac{\arccos (-\alpha)}{n}$, we have

$$
T_{n}\left(x_{2}-\left(x-\frac{\pi}{n}\right)\right) \leqslant \cos n x+\alpha \leqslant 0
$$

and therefore

$$
\left|T_{n}\left(x_{2}-\left(x-\frac{\pi}{n}\right)\right)\right| \geqslant|\cos n x+\alpha|
$$

Further, let

$$
A_{1}:=\left(x_{1}-\frac{\arccos (-\alpha)}{n}, x_{1}+\frac{\arccos (-\alpha)}{n}\right), A_{2}:=\left(x_{2}-\left(\frac{\pi}{n}-\frac{\arccos (-\alpha)}{n}\right), x_{2}+\left(\frac{\pi}{n}-\frac{\arccos (-\alpha)}{n}\right)\right) .
$$

Since $\left|x_{1}-x_{2}\right| \geqslant \frac{\pi}{n}$, then $A_{1} \cap A_{2}=\emptyset$. Moreover, $\left|A_{1}\right|+\left|A_{2}\right|=2 \pi / n \leqslant d$ and for $\gamma>0$

$$
\begin{aligned}
\left|\left\{x \in[0, d):\left|T_{n}(x)\right|>\gamma\right\}\right| & \geqslant\left|\left\{x \in A_{1} \cup A_{2}:\left|T_{n}(x)\right|>\gamma\right\}\right| \\
& =\left|\left\{x \in A_{1}:\left|T_{n}(x)\right|>\gamma\right\}\right|+\left|\left\{x \in A_{2}:\left|T_{n}(x)\right|>\gamma\right\}\right| .
\end{aligned}
$$

Then, making use of (2.9) and (2.10) and translation invariance of the distribution function, we have for $\gamma>0$

$$
\begin{aligned}
\left|\left\{x \in[0, d):\left|T_{n}(x)\right|>\gamma\right\}\right| \geqslant & \left|\left\{x \in\left(-\frac{\arccos (-\alpha)}{n}, \frac{\arccos (-\alpha)}{n}\right):|\cos n x+\alpha|>\gamma\right\}\right| \\
& +\left|\left\{x \in\left(\frac{\arccos (-\alpha)}{n}, \frac{2 \pi}{n}-\frac{\arccos (-\alpha)}{n}\right):|\cos n x+\alpha|>\gamma\right\}\right| \\
= & \left|\left\{x \in\left(0, \frac{2 \pi}{n}\right):|\cos n x+\alpha|>\gamma\right\}\right| .
\end{aligned}
$$

Therefore, for $\alpha \in[-1,1]$ we get the required inequality $(2.5)$ for $0 \leqslant \beta<\frac{2 \pi}{n}$.

Let now $\alpha>1$ and therefore $\xi>0$. Applying (2.5) to $T_{n}-\xi$, it follows that

$$
\left(T_{n}-\xi\right)^{\star}(\beta) \geqslant(\cos n x+1)^{\star}(\beta), \quad 0 \leqslant \beta<\frac{2 \pi}{n} .
$$

Taking into account that

$$
T_{n}^{\star}(\beta)=\left(T_{n}-\xi\right)^{\star}(\beta)+\xi
$$

and

$$
(\cos n x+\alpha)^{\star}(\beta)=(\cos n x+\xi+1)^{\star}(\beta)=(\cos n x+1)^{\star}(\beta)+\xi,
$$

we get (2.5) for $\alpha>1$. For $\alpha<-1$ it is enough to consider $-T_{n}$.

Lemma 2.4. Let $n \in \mathbb{N}$ and $0 \leqslant \beta<\frac{2 \pi}{n}$. Then

(a)

(b)

$$
\sup _{0 \leqslant \alpha \leqslant 1} \frac{1+\alpha}{(\cos n x+\alpha)^{\star}(\beta)} \leqslant \frac{1+\sin ^{2} \frac{n \beta}{4}}{1-\sin ^{2} \frac{n \beta}{4}}
$$

$$
\inf _{0 \leqslant \alpha<\infty}(\cos n x+\alpha)^{\star}(\beta) \geqslant \cos ^{2} \frac{n \beta}{4} ;
$$

(c)

$$
\left(\cos n x+\frac{1-\cos \frac{n \beta}{2}}{2}\right)^{\star}(\beta)=\cos ^{2} \frac{n \beta}{4}
$$


Proof. Let $\cos \frac{n \beta}{2}+\alpha \geqslant 1-\alpha$, i.e., $\alpha \geqslant \frac{\left(1-\cos \frac{n \beta}{2}\right)}{2}$ (see Figure 1 showing the graph of the function $f(x)=$ $|\cos n x+\alpha|)$.

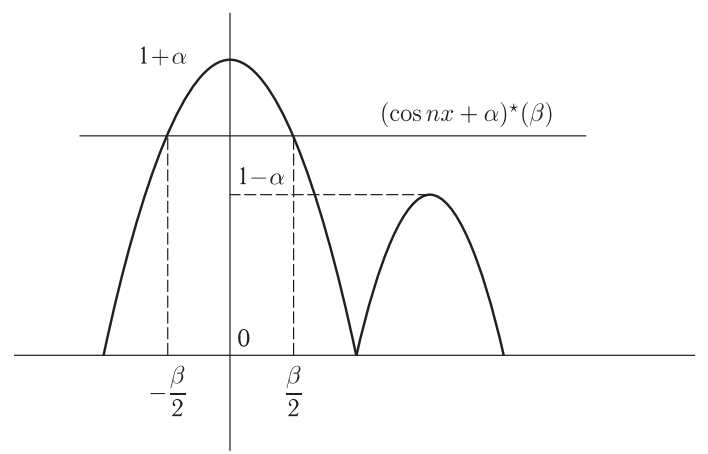

FIGURE 1.

Then

$$
(\cos n x+\alpha)^{\star}(\beta)=\cos \frac{n \beta}{2}+\alpha .
$$

Note that the function

$$
\varphi(\alpha)=\frac{1+\alpha}{\cos \frac{n \beta}{2}+\alpha}
$$

is monotone with respect to $\alpha$ and therefore

$$
\sup _{0 \leqslant \alpha \leqslant 1} \frac{1+\alpha}{(\cos n x+\alpha)^{\star}(\beta)}=\sup _{0 \leqslant \alpha \leqslant\left(1-\cos \frac{n \beta}{2}\right) / 2} \frac{1+\alpha}{(\cos n x+\alpha)^{\star}(\beta)} .
$$

Next let $0 \leqslant \alpha \leqslant \frac{1-\cos \frac{n \beta}{2}}{2}$, then

$$
(\cos n x+\alpha)^{\star}(\beta)=\frac{\cos \frac{n u}{2}+\cos \frac{n v}{2}}{2},
$$

where $0 \leqslant u \leqslant v \leqslant \beta$ satisfy the following equations

$$
\left\{\begin{array}{l}
u+v=\beta \\
\cos \frac{n u}{2}-\alpha=\cos \frac{n v}{2}+\alpha
\end{array}\right.
$$

(see Figure 2).

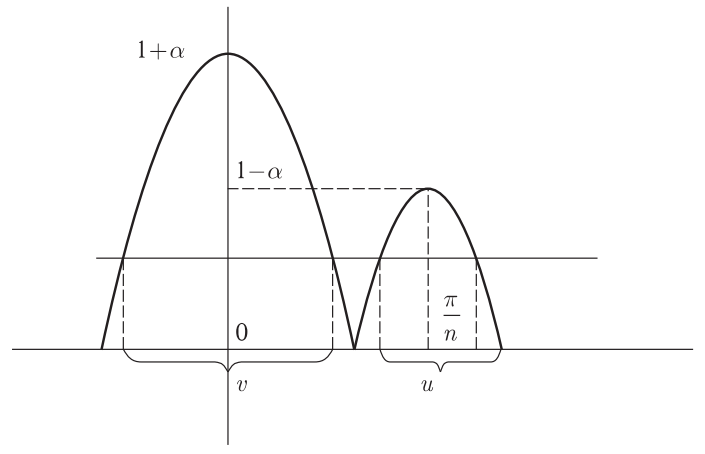

Figure 2.

Hence,

$$
\frac{1+\alpha}{(\cos n x+\alpha)^{\star}(\beta)}=\frac{2+\cos \frac{n u}{2}-\cos \frac{n(\beta-u)}{2}}{\cos \frac{n u}{2}+\cos \frac{n(\beta-u)}{2}} .
$$


Let us find the maximum of the right-hand side for $u \in[0, \beta / 2]$. Take

$$
f(z)=\frac{2+\cos z-\cos (a-z)}{\cos z+\cos (a-z)}, \quad z \in[0, a / 2),
$$

where $0 \leqslant a<\pi$. Then

$$
f^{\prime}(z)=\frac{-2 \sin a-2 \sin (a-z)+2 \sin z}{(\cos z+\cos (a-z))^{2}}<0, \quad z \in[0, a / 2) .
$$

Therefore, $f(z)$ is non-increasing on $\left[0, \frac{a}{2}\right)$ and has a maximum at $z=0$. Then, by (2.12),

$$
\begin{aligned}
\sup _{0 \leqslant \alpha \leqslant 1} \frac{1+\alpha}{(\cos n x+\alpha)^{\star}(\beta)} & =\max _{0 \leqslant u \leqslant \frac{\beta}{2}} \frac{2+\cos \frac{n u}{2}-\cos \frac{n(\beta-u)}{2}}{\cos \frac{n u}{2}+\cos \frac{n(\beta-u)}{2}} \\
& =\frac{3-\cos \frac{n \beta}{2}}{1+\cos \frac{n \beta}{2}}=\frac{1+\sin ^{2} \frac{n \beta}{4}}{1-\sin ^{2} \frac{n \beta}{4}}
\end{aligned}
$$

i.e., (a) is verified.

To prove (b), let $\alpha>1$ and then

$$
(\cos n x+\alpha)^{\star}=(\cos n x+1)^{\star}+\alpha-1>(\cos n x+1)^{\star} .
$$

Therefore, in (b), infimum is attained for $0 \leqslant \alpha \leqslant 1$. Further we remark that for $\alpha>\frac{1-\cos \frac{n \beta}{2}}{2}$ we have

$$
(\cos n x+\alpha)^{\star}(\beta)=\cos \frac{n \beta}{2}+\alpha,
$$

which is the non-decreasing function with respect to $\alpha$. Then

$$
\inf _{0 \leqslant \alpha<\infty}(\cos n x+\alpha)^{\star}(\beta) \geqslant \inf _{0 \leqslant \alpha \leqslant\left(1-\cos \frac{n \beta}{2}\right) / 2}(\cos n x+\alpha)^{\star}(\beta) .
$$

Next, for $0 \leqslant \alpha \leqslant\left(1-\cos \frac{n \beta}{2}\right) / 2$, we have

$$
(\cos n x+\alpha)^{\star}(\beta)=\frac{\cos \frac{n u}{2}+\cos \frac{n v}{2}}{2}, \quad 0 \leqslant u \leqslant v \leqslant \beta,
$$

where $u, v$, and $\alpha$ are related by (2.13). Taking into account that

$$
\min _{0 \leqslant u \leqslant \frac{\beta}{2}} \frac{\cos \frac{n(\beta-u)}{2}+\cos \frac{n u}{2}}{2}=\cos ^{2} \frac{n \beta}{4},
$$

we get (b).

To prove (c), we just use (2.11):

$$
(\cos n x+\alpha)^{\star}(\beta)=\cos \frac{n \beta}{2}+\frac{\left(1-\cos \frac{n \beta}{2}\right)}{2}=\cos ^{2} \frac{n \beta}{4} .
$$

\section{Sharp Remez inequalities}

First, we note that the classical Remez inequality can be written as an inequality in terms of rearrangements (see also $[\mathrm{DP}]$ ).

Remark 3.1. Let $\Omega$ be a measurable subset of $\mathbb{R}, f \in L_{\infty}(\Omega)$ and a right continuous function $C(\cdot)$ be nondecreasing. Then the following statements are equivalent:

$$
f^{*}(0) \leqslant C(\beta) f^{*}(\beta), \quad \beta>0 ;
$$

$$
\begin{gathered}
\|f\|_{L_{\infty}(\Omega)} \leqslant C(|B|)\|f\|_{L_{\infty}(\Omega \backslash B)} \text { for any measurable set } B \subset \Omega ; \\
\quad \text { if } \quad|\{x \in \Omega:|f(x)|>1\}|<\beta, \text { then }\|f\|_{L_{\infty}(\Omega)} \leqslant C(\beta) .
\end{gathered}
$$


Proof. Let us verify that $(3.1) \Rightarrow(3.2) \Rightarrow(3.3) \Rightarrow(3.1)$. First assume that (3.1) holds. Let $B$ be a subset of $\Omega$ such that $|B|=\beta$. Note that for any $\varepsilon>0$

$$
A=\left|\left\{x \in \Omega:|f(x)| \geqslant f^{*}(\beta+\varepsilon)\right\}\right| \geqslant(\beta+\varepsilon) .
$$

Then

and

$$
\left|\left\{x \in \Omega:|f(x)| \geqslant f^{*}(\beta+\varepsilon)\right\} \backslash B\right|>0
$$

Using (3.1), we get

$$
\|f\|_{L_{\infty}(\Omega \backslash B)} \geqslant\|f\|_{L_{\infty}(A \backslash B)} \geqslant f^{*}(\beta+\varepsilon) .
$$

$$
\|f\|_{L_{\infty}(\Omega)}=f^{*}(0) \leqslant C(\beta+\varepsilon) f^{*}(\beta+\varepsilon) \leqslant C(\beta+\varepsilon)\|f\|_{L_{\infty}(\Omega \backslash B)},
$$

and therefore (3.2) holds.

Let now (3.2) hold. Denoting $B:=\{x \in \Omega:|f(x)|>1\}$ and assuming $|B|<\beta$, we have $|f(x)| \leqslant 1$ for any $x \in \Omega \backslash B$. Then (3.2) gives

$$
\|f\|_{L_{\infty}(\Omega)} \leqslant C(|B|)\|f\|_{L_{\infty}(\Omega \backslash B)} \leqslant C(|B|) \leqslant C(\beta) .
$$

Assuming (3.3), consider $\beta>0$ and $\psi(x)=\frac{f(x)}{f^{*}(\beta)}$, we get

$$
|\{x \in \Omega:|\psi(x)|>1\}|=\left|\left\{x \in \Omega:|f(x)|>f^{*}(\beta)\right\}\right| \leqslant \beta<\beta+\varepsilon .
$$

By (3.3) we have $\|\psi\|_{L_{\infty}(\Omega)} \leqslant C(\beta+\varepsilon)$ for any $\varepsilon>0$. Since $C(\cdot)$ is right continuous, $\|\psi\|_{L_{\infty}(\Omega)} \leqslant C(\beta)$, i.e., (3.1) follows.

Thus, to investigate the Remez inequality, one can use its rearrangement form

$$
T_{n}^{*}(0) \leqslant C(n, \beta) T_{n}^{*}(\beta), \quad \beta>0 .
$$

Note that dealing with polynomials with the minimal periods less than $2 \pi$ allows us to decrease the constant $C(n, \beta)$. Indeed, if $d=2 \pi / m$ is a period of $T_{n}$, then by Lemma $2.1, T_{n}(x / m)$ is a polynomial of degree $n / m$ and

$$
\bar{T}_{\frac{n}{m}}(x):=T_{n}(x / m), \quad \bar{T}_{\frac{n}{m}}^{*}(\beta)=T_{n}^{*}(\beta), \quad \beta>0 .
$$

Then inequality (3.4) is true with the constant $C\left(\frac{n}{m}, \beta\right)$.

To overcome this, we are going to study Remez's inequality with the $\star$-rearrangement, i.e.,

$$
T_{n}^{\star}(0) \leqslant C(n, \beta) T_{n}^{\star}(\beta), \quad \beta>0 .
$$

Let us now recall that $\mathfrak{N}_{n}$ and $\mathfrak{M}_{n}$ are the sets of complex and real polynomials of degree at most $n$, respectively. The next remark shows that to study the Remez inequality in the form (3.4) or in the form (3.5), it suffices to deal with real polynomials.

Remark 3.2. Let $0 \leqslant \beta<2 \pi$. Then

$$
\sup _{T_{n} \in \mathfrak{N}_{n}} \frac{T_{n}^{*}(0)}{T_{n}^{*}(\beta)}=\sup _{T_{n} \in \mathfrak{M}_{n}} \frac{T_{n}^{*}(0)}{T_{n}^{*}(\beta)}
$$

and

$$
\sup _{T_{n} \in \mathfrak{N}_{n}} \frac{T_{n}^{\star}(0)}{T_{n}^{\star}(\beta)}=\sup _{T_{n} \in \mathfrak{M}_{n}} \frac{T_{n}^{\star}(0)}{T_{n}^{\star}(\beta)}
$$

Proof. It is clear that

$$
\sup _{T_{n} \in \mathfrak{N}_{n}} \frac{T_{n}^{*}(0)}{T_{n}^{*}(\beta)} \geqslant \sup _{T_{n} \in \mathfrak{M}_{n}} \frac{T_{n}^{*}(0)}{T_{n}^{*}(\beta)}
$$

Let now $T_{n} \in \mathfrak{N}_{n}$ and $\left|T_{n}\left(x_{0}\right)\right|=\max _{x \in \mathbb{T}}\left|T_{n}(x)\right|$. Suppose $\alpha=\frac{T_{n}\left(x_{0}\right)}{\left|T_{n}\left(x_{0}\right)\right|}$, then $\operatorname{Re}\left(\bar{\alpha} T_{n}\right) \in \mathfrak{M}_{n}$ and

$$
\max _{x \in \mathbb{T}}\left|\operatorname{Re}\left(\bar{\alpha} T_{n}\right)(x)\right|=\left|T_{n}\left(x_{0}\right)\right|=T_{n}^{*}(0) .
$$


Further, since $\left|\operatorname{Re}\left(\bar{\alpha} T_{n}(x)\right)\right| \leqslant\left|T_{n}(x)\right|$, we get

$$
\frac{T_{n}^{*}(0)}{T_{n}^{*}(\beta)}=\frac{\left(\operatorname{Re}\left(\bar{\alpha} T_{n}\right)\right)^{*}(0)}{T_{n}^{*}(\beta)} \leqslant \frac{\left(\operatorname{Re}\left(\bar{\alpha} T_{n}\right)\right)^{*}(0)}{\left(\operatorname{Re}\left(\bar{\alpha} T_{n}\right)\right)^{*}(\beta)} \leqslant \sup _{T_{n} \in \mathfrak{M}_{n}} \frac{T_{n}^{*}(0)}{T_{n}^{*}(\beta)}
$$

and (3.6) follows. Similarly, we can show (3.7).

3.1. Sharp Remez inequalities in terms of rearrangements. The main result of this section is the following sharp Remez type inequalities.

Theorem 3.3. Let $n, k \in \mathbb{N}$ and $0 \leqslant \beta<\frac{2 \pi}{n}$. Then

$$
\sup _{T_{n} \in \mathfrak{M}_{n}} \frac{T_{n}^{\star}(0)}{T_{n}^{\star}(\beta)}=1+2 \tan ^{2} \frac{n \beta}{4}
$$

and

$$
\sup _{T_{n} \in \mathfrak{M}_{n}} \frac{\left\|n^{-k} T_{n}^{(k)}\right\|_{L_{\infty}}}{T_{n}^{\star}(\beta)}=1+\tan ^{2} \frac{n \beta}{4} .
$$

Proof. To prove (3.8), we define two subsets of $\mathfrak{M}_{n}$ :

$$
\mathfrak{M}_{n}^{0}=\left\{T_{n} \in \mathfrak{M}_{n}: \quad \max _{x} T_{n}(x)-\min _{x} T_{n}(x)=2\right\}
$$

and

$$
\mathfrak{M}_{n}^{1}=\left\{T_{n} \in \mathfrak{M}_{n}^{0}: \quad 1 \leqslant \max _{x} T_{n}(x) \leqslant 2\right\} .
$$

Note that for any $T_{n} \neq$ const there exists $\gamma>0$ such that $\gamma T_{n} \in \mathfrak{M}_{n}^{0}$. Then it is clear that

$$
\sup _{T_{n} \in \mathfrak{M}_{n}} \frac{T_{n}^{\star}(0)}{T_{n}^{\star}(\beta)}=\sup _{T_{n} \in \mathfrak{M}_{n}^{0}} \frac{T_{n}^{\star}(0)}{T_{n}^{\star}(\beta)} .
$$

Let us now show that

$$
\sup _{T_{n} \in \mathfrak{M}_{n}^{0}} \frac{T_{n}^{\star}(0)}{T_{n}^{\star}(\beta)}=\sup _{T_{n} \in \mathfrak{M}_{n}^{1}} \frac{T_{n}^{\star}(0)}{T_{n}^{\star}(\beta)} .
$$

Suppose that $T_{n} \in \mathfrak{M}_{n}^{0}$ but $T_{n} \notin \mathfrak{M}_{n}^{1}$ and $-T_{n} \notin \mathfrak{M}_{n}^{1}$. Denoting $\Xi:=\max T_{n}(x)$, note that $\Xi>2$. Indeed, assume $0 \leqslant \Xi<1$, then it is clear $-T_{n} \in \mathfrak{M}_{n}^{1}$, which contradicts the choice of $T_{n}$. The case $\Xi<0$ can be reduced to the case $\Xi>0$ by taking $-T_{n}$.

Therefore, $\Xi>2$ and $\xi:=\min T_{n}(x)>0$. Then for $P_{n}(x):=T_{n}(x)-\xi \geqslant 0$ we have

$$
P_{n}^{\star}(\beta)=T_{n}^{\star}(\beta)-\xi, \quad \beta \geqslant 0 .
$$

On the other hand, $P_{n} \in \mathfrak{M}_{n}^{1}$,

$$
\frac{T_{n}^{\star}(0)}{T_{n}^{\star}(\beta)}=\frac{\left(T_{n}^{\star}(0)-\xi\right)^{\star}+\xi}{\left(T_{n}(\beta)-\xi\right)^{\star}+\xi}=\frac{P_{n}^{\star}(0)+\xi}{P_{n}^{\star}(\beta)+\xi} \leqslant \frac{P_{n}^{\star}(0)}{P_{n}^{\star}(\beta)},
$$

because of $P_{n}^{\star}(0) \geqslant P_{n}^{\star}(\beta)$ and $\xi>0$. Hence, we get

$$
\sup _{T_{n} \in \mathfrak{M}_{n}} \frac{T_{n}^{\star}(0)}{T_{n}^{\star}(\beta)}=\sup _{T_{n} \in \mathfrak{M}_{n}^{1}} \frac{T_{n}^{\star}(0)}{T_{n}^{\star}(\beta)} .
$$

Therefore, it suffices to verify (3.8) on the class $\mathfrak{M}_{n}^{1}$.

Let further $T_{n} \in \mathfrak{M}_{n}^{1}$, then by Lemma 2.3 ,

$$
T_{n}^{\star}(\beta) \geqslant(\cos n x+\alpha)^{\star}(\beta),
$$

where $0 \leqslant \alpha=\Xi-1 \leqslant 1$. Then

and therefore,

$$
\frac{T^{\star}(0)}{T^{\star}(\beta)} \leqslant \frac{\Xi}{(\cos n x+\alpha)^{\star}(\beta)}=\frac{1+\alpha}{(\cos n x+\alpha)^{\star}(\beta)}
$$

$$
\sup _{T_{n} \in \mathfrak{M}_{n}^{1}} \frac{T^{\star}(0)}{T^{\star}(\beta)} \leqslant \sup _{0 \leqslant \alpha \leqslant 1} \frac{1+\alpha}{(\cos n x+\alpha)^{\star}(\beta)} .
$$


Making use of Lemma 2.4(a), we get

$$
\sup _{T_{n} \in \mathfrak{M}_{n}} \frac{T_{n}^{\star}(0)}{T_{n}^{\star}(\beta)}=\sup _{T_{n} \in \mathfrak{M}_{n}^{1}} \frac{T^{\star}(0)}{T^{\star}(\beta)} \leqslant \frac{1+\sin ^{2} \frac{\beta n}{4}}{1-\sin ^{2} \frac{\beta n}{4}}=1+2 \tan ^{2} \frac{\beta n}{4},
$$

where $0 \leqslant \beta<\frac{2 \pi}{n}$. The constant in (3.8) is sharp, take

$$
T_{n}(x)=\cos n x+\frac{1-\cos \frac{n \beta}{2}}{2}
$$

and use Lemma 2.4(c).

Next, let us show the accuracy of (3.9). Remark that

$$
\sup _{T_{n} \in \mathfrak{M}_{n}} \frac{\left\|n^{-k} T_{n}^{(k)}\right\|_{L_{\infty}}}{T_{n}^{\star}(\beta)}=\sup _{T_{n} \in \mathfrak{M}_{n}^{1}} \frac{\left\|n^{-k} T_{n}^{(k)}\right\|_{L_{\infty}}}{T_{n}^{\star}(\beta)} .
$$

Let $T_{n} \in \mathfrak{M}_{n}^{1}$, then $1 \leqslant \Xi \leqslant 2, \max _{x}\left|T_{n}(x)-(\Xi-1)\right|=1$. Using Bernstein's inequality, we have

$$
\sup _{T_{n} \in \mathfrak{M}_{n}^{1}} \frac{\left\|n^{-k} T_{n}^{(k)}\right\|_{L_{\infty}}}{T_{n}^{\star}(\beta)}=\sup _{T_{n} \in \mathfrak{M}_{n}^{1}} \frac{\left\|n^{-k}\left(T_{n}-(\Xi-1)\right)^{(k)}\right\|_{L_{\infty}}}{T_{n}^{\star}(\beta)} \leqslant \sup _{T_{n} \in \mathfrak{M}_{n}^{1}} \frac{1}{T_{n}^{\star}(\beta)} .
$$

By Lemma 2.3,

$$
\sup _{T_{n} \in \mathfrak{M}_{n}^{1}} \frac{\left\|n^{-k} T_{n}^{(k)}\right\|_{L_{\infty}}}{T_{n}^{\star}(\beta)} \leqslant \sup _{T_{n} \in \mathfrak{M}_{n}^{1}} \frac{1}{(\cos n x+\Xi-1)^{\star}(\beta)}=\frac{1}{\inf _{0 \leqslant c<\infty}(\cos n x+c)^{\star}(\beta)} .
$$

Also, taking into account Lemma 2.4 (b), we get

$$
\sup _{T_{n} \in \mathfrak{M}_{n}} \frac{\left\|n^{-k} T_{n}^{(k)}\right\|_{L_{\infty}}}{T_{n}^{\star}(\beta)}=\sup _{T_{n} \in \mathfrak{M}_{n}^{1}} \frac{\left\|n^{-k} T_{n}^{(k)}\right\|_{L_{\infty}}}{T_{n}^{\star}(\beta)} \leqslant \frac{1}{\cos ^{2} \frac{n \beta}{4}} .
$$

Sharpness in (3.9) can be verified by considering the function (3.12); see Lemma 2.4(c).

Note that first applying Bernstein's inequality and then Remez's inequality (3.8) does not give the sharp constant in the Remez-Bernstein inequality (3.9).

Corollary 3.4. Let $n, k \in \mathbb{N}, T_{n} \in \mathfrak{M}_{n}, 2 \pi / m$ be the minimal period of $T_{n}$, and $0 \leqslant \beta<\frac{2 \pi m}{n}$. Then

$$
T_{n}^{*}(0) \leqslant\left(1+2 \tan ^{2} \frac{n \beta}{4 m}\right) T_{n}^{*}(\beta)
$$

and

$$
\left\|n^{-k} T_{n}^{(k)}\right\|_{L_{\infty}} \leqslant\left(1+\tan ^{2} \frac{n \beta}{4 m}\right) T_{n}^{*}(\beta) .
$$

Inequalities (3.13) and (3.14) are sharp; they become equalities for $T_{n}(x)=\cos n x+\frac{1}{2}\left(1-\cos \frac{\beta}{2}\right)$.

Proof. Let $0 \leqslant \beta<\frac{2 \pi m}{n}$ and $k \in \mathbb{N}$. Then Theorem 3.3, where (3.8) and (3.9) are applied at the point $\beta / m$, and Lemma 2.1(e) give

and

$$
T_{n}^{*}(0)=T_{n}^{\star}(0) \leqslant\left(1+2 \tan ^{2} \frac{n \beta}{4 m}\right) T_{n}^{\star}(\beta / m)=\left(1+2 \tan ^{2} \frac{n \beta}{4 m}\right) T_{n}^{*}(\beta)
$$

$$
\left\|n^{-k} T_{n}^{(k)}\right\|_{L_{\infty}} \leqslant\left(1+\tan ^{2} \frac{n \beta}{4 m}\right) T_{n}^{\star}(\beta / m)=\left(1+\tan ^{2} \frac{n \beta}{4 m}\right) T_{n}^{*}(\beta) .
$$

The next result provides the asymptotics of the sharp constant $\mathcal{C}(n, \beta)$ in the Remez inequality.

Corollary 3.5. We have

$$
\mathcal{C}(n, \beta)=1+\frac{(n \beta)^{2}}{8}+O\left(\beta^{4}\right)
$$

and

$$
\mathcal{C}(1, \beta)=1+2 \tan ^{2} \frac{\beta}{4}
$$


Proof. It follows from (1.4) and (1.7) that

$$
A_{n}(\beta):=\frac{1}{2}\left(\tan ^{2 n}\left(\frac{2 \pi-\beta}{8}\right)+\cot ^{2 n}\left(\frac{2 \pi-\beta}{8}\right)\right) \leqslant \mathcal{C}(n, \beta) \leqslant 1+2 \tan ^{2} \frac{n \beta}{4}, \quad 0<\beta<\frac{2 \pi}{n} .
$$

Let $x=\beta / 8$. Then

$$
\begin{aligned}
A_{n}(x)-1= & \frac{1}{2}\left[\left(\cot ^{2 n}(\pi / 4-x)-1\right)+\left(\tan ^{2 n}(\pi / 4-x)-1\right)\right] \\
= & \frac{1}{2}\left[\left(\frac{\cos (\pi / 2-2 x)}{\sin ^{2}(\pi / 4-x)} \sum_{k=0}^{n-1} \cot ^{2 k}(\pi / 4-x)\right)-\left(\frac{\cos (\pi / 2-2 x)}{\cos ^{2}(\pi / 4-x)} \sum_{k=0}^{n-1} \tan ^{2 k}(\pi / 4-x)\right)\right] \\
= & \frac{\sin 2 x}{2}\left[\frac{1}{\sin ^{2}(\pi / 4-x)} \sum_{k=0}^{n-1}\left(\cot ^{2 k}(\pi / 4-x)-1\right)-\frac{1}{\cos ^{2}(\pi / 4-x)} \sum_{k=0}\left(\tan ^{2 k}(\pi / 4-x)-1\right)\right. \\
& \left.+\frac{n}{\sin ^{2}(\pi / 4-x)}-\frac{n}{\cos ^{2}(\pi / 4-x)}\right] \\
= & \frac{\sin 2 x}{2}\left[\frac{1}{\sin ^{2}(\pi / 4-x)} \sum_{k=0}^{n-1} \frac{\cos (\pi / 2-2 x)}{\sin ^{2}(\pi / 4-x)} \sum_{m=0}^{k-1} \cot ^{2 m}(\pi / 4-x)\right. \\
& \left.+\frac{1}{\cos ^{2}(\pi / 4-x)} \sum_{k=0}^{n-1} \frac{\cos ^{2}(\pi / 2-2 x)}{\cos ^{2}(\pi / 4-x)} \sum_{m=0}^{k-1} \tan ^{2 m}(\pi / 4-x)+\frac{n \cos (\pi / 2-2 x)}{\sin ^{2}(\pi / 4-x) \cos ^{2}(\pi / 4-x)}\right] \\
= & \frac{\sin ^{2} 2 x}{2}\left[\frac{1}{\sin ^{2}(\pi / 4-x)} \sum_{k=0}^{n-1} \frac{1}{\sin ^{2}(\pi / 4-x)} \sum_{m=0}^{k-1} \cot ^{2 m}(\pi / 4-x)\right. \\
& \left.+\frac{1}{\cos ^{2}(\pi / 4-x)} \sum_{k=0}^{n-1} \frac{1}{\cos ^{2}(\pi / 4-x)} \sum_{m=0}^{k-1} \tan ^{2 m}(\pi / 4-x)+\frac{\sin ^{2}(\pi / 4-x) \cos ^{2}(\pi / 4-x)}{n}\right]
\end{aligned}
$$

Since $A_{n}(x)$ is even, we get

$$
A_{n}(x)-1=\frac{\sin ^{2} 2 x}{2\left(\frac{\sqrt{2}}{2}\right)^{4}}\left(2 \sum_{k=1}^{n-1} k+n+O\left(x^{2}\right)\right)=2 \sin ^{2} \frac{\beta}{4}\left(n^{2}+O\left(\beta^{2}\right)\right)=\frac{(n \beta)^{2}}{8}+O\left(\beta^{4}\right)
$$

or

$$
A_{n}(\beta)=1+\frac{(n \beta)^{2}}{8}+O\left(\beta^{4}\right)
$$

On the other hand,

$$
1+2 \tan ^{2} \frac{n \beta}{4}=1+\frac{(n \beta)^{2}}{8}+O\left(\beta^{4}\right)
$$

which implies (3.15).

Further, (3.16) follows from $(n=1$ and $x=\beta / 8)$

$$
\begin{aligned}
A_{n}(\beta)-1 & =\frac{1}{2}\left[\left(\cot ^{2}(\pi / 4-x)-1\right)+\left(\tan ^{2}(\pi / 4-x)-1\right)\right] \\
& =\frac{\sin ^{2} 2 x}{2}\left[\frac{1}{\sin ^{2}(\pi / 4-x) \cos ^{2}(\pi / 4-x)}\right]=2 \tan ^{2} 2 x=2 \tan ^{2} \frac{\beta}{4} .
\end{aligned}
$$

Remark 3.6. Corollary 3.4 yields the following Remez inequality with the constant written in the exponential form. If $d=2 \pi / m$ is the minimal period of $T_{n} \in \mathfrak{M}_{n}$, then

$$
\left\|T_{n}\right\|_{L_{\infty}[0,2 \pi)} \leqslant \exp \left(\frac{(n \beta)^{2}}{2 m^{2}}\right)\left\|T_{n}\right\|_{L_{\infty}([0,2 \pi) \backslash B)}, \quad|B|=\beta<\frac{m \pi}{n}
$$

compare with the known bound $\exp (n \beta \min \{2,(1+1.75 \beta)\})$, cf. (1.4). This follows from (3.13) and the inequality $1+2 \tan ^{2} x / 4 \leqslant \exp \left(\frac{x^{2}}{2}\right)$ for $0<x<\pi$.

Next we provide the following result on the Remez inequality in $L_{p}$ (see also [Er2] and [EMN]). 
Corollary 3.7. Let $p \in(0, \infty)$ and $2 \pi / m$ be a minimal period of $T_{n} \in \mathfrak{M}_{n}$. Then

where $|B|<\frac{\pi m}{n}$.

$$
\int_{0}^{2 \pi}\left|T_{n}(t)\right|^{p} d t \leqslant\left[1+\left(1+2 \tan ^{2} \frac{n|B|}{2 m}\right)^{p}\right] \int_{\mathbb{T} \backslash B}\left|T_{n}(t)\right|^{p} d t,
$$

Proof. Using Corollary 3.4, by (3.13), we get

$$
\begin{aligned}
\int_{B}\left|T_{n}(x)\right|^{p} d x & \leqslant \int_{0}^{|B|}\left(T_{n}^{*}(t)\right)^{p} d t \\
& \leqslant|B|\left(1+2 \tan ^{2} \frac{n|B|}{2 m}\right)^{p}\left(T_{n}^{*}(2|B|)\right)^{p} \\
& \leqslant\left(1+2 \tan ^{2} \frac{n|B|}{2 m}\right)^{p} \int_{|B|}^{2|B|}\left(T_{n}^{*}(t)\right)^{p} d t \\
& \leqslant\left(1+2 \tan ^{2} \frac{n|B|}{2 m}\right)^{p} \int_{|B|}^{2 \pi}\left(T_{n}^{*}(t)\right)^{p} d t \\
& \leqslant\left(1+2 \tan ^{2} \frac{n|B|}{2 m}\right)^{p} \int_{\mathbb{T} \backslash B}\left|T_{n}(x)\right|^{p} d x .
\end{aligned}
$$

3.2. Sharp Remez inequalities in terms of averages. Remez's inequality allows us to control how fast polynomials of degree $n$ change on the period. Another inequality that characterizes such properties of polynomials is the following one:

$$
\left\|T_{n}\right\|_{L_{\infty}} \leqslant C(n, \beta) \overline{T_{n}(\beta, M)} .
$$

Let $[a, b]$ be an interval of $\mathbb{T}$ of the measure $\leqslant d$. We say that $Q$ is a harmonic interval if

$$
Q=\bigcup_{i=0}^{r}([a, b]+i d), \quad r \in \mathbb{N}
$$

(see e.g. $[\mathrm{Nu}]$ ). By $M_{\text {h.int. }}$ we denote the collection of all harmonic intervals in $\mathbb{T}$. Let also $M_{\text {int }}$. be the collection of all intervals in $\mathbb{T}$.

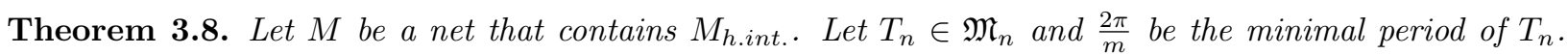
Then

$$
\left\|T_{n}\right\|_{L_{\infty}} \leqslant \frac{n \beta}{2 m \sin \frac{n \beta}{2 m}} \overline{T_{n}(\beta, M)}, \quad 0<\beta<\frac{\pi m}{n},
$$

and

$$
\left\|T_{n}\right\|_{L_{\infty}} \leqslant \frac{n \beta}{2 \sin \frac{n \beta}{2}} \overline{T_{n}\left(\beta, M_{i n t .}\right)}, \quad 0<\beta<\frac{\pi}{n} .
$$

Inequalities (3.18) and (3.19) are sharp; they become equalities for $T_{n}(x)=\cos n x$.

Remark 3.9. An analogue of (3.19) for a net $M$ that contains $M_{i n t .}$ also holds, i.e.,

$$
\left\|T_{n}\right\|_{L_{\infty}} \leqslant \frac{n \beta}{2 \sin \frac{n \beta}{2}} \overline{T_{n}(\beta, M)}, \quad 0<\beta<\frac{\pi}{n} .
$$

However, unlike (3.18) this inequality is not sharp.

Proof of Theorem 3.8. Since $\frac{\left\|T_{n}\right\|_{L_{\infty}}}{\overline{T_{n}(\beta, M)}}$ is invariant under multiplication by $\gamma \in \mathbb{R} \backslash\{0\}$, we get

$$
\sup _{T_{n} \in \mathfrak{M}_{n}} \frac{\left\|T_{n}\right\|_{L_{\infty}}}{\overline{T_{n}(\beta, M)}}=\sup _{T_{n} \in \mathfrak{M}_{n}^{0}} \frac{\left\|T_{n}\right\|_{L_{\infty}}}{\overline{T_{n}(\beta, M)}} .
$$

Let then $T_{n} \in \mathfrak{M}_{n}^{0}$. Without loss of generality we assume that $\Xi=\max _{x \in[-\pi, \pi)} T_{n}(x)=T_{n}(0) \geqslant 1$. Then Lemma 2.2 implies

$$
T_{n}(x) \geqslant \cos n x+(\Xi-1), \quad x \in\left[-\frac{\pi}{2 n}, \frac{\pi}{2 n}\right] .
$$


This and $\frac{2 \pi}{m}$-periodicity of $T_{n}$ give for $\beta \in(0, \pi m / n)$

$$
\begin{aligned}
\frac{1}{\beta} \sum_{i=0}^{m-1} \int_{-\frac{\beta}{2 m}+\frac{2 \pi i}{m}}^{\frac{\beta}{2 m}+\frac{2 \pi i}{m}} T_{n}(x) d x & =\frac{m}{\beta} \int_{-\frac{\beta}{2 m}}^{\frac{\beta}{2 m}} T_{n}(x) d x \\
& \geqslant \frac{m}{\beta} \int_{-\frac{\beta}{2 m}}^{\frac{\beta}{2 m}}(\cos n x+\Xi-1) d x=\frac{2 m \sin \frac{n \beta}{2 m}}{n \beta}+\Xi-1 \geqslant 0 .
\end{aligned}
$$

Hence,

$$
\sup _{\substack{e \in M_{h . i n t .} \\|e|=\beta}} \frac{1}{|e|}\left|\int_{e} T_{n}(y) d y\right| \geqslant \frac{1}{\beta} \sum_{i=0}^{m-1} \int_{-\frac{\beta}{2 m}+\frac{2 \pi i}{m}}^{\frac{\beta}{2 m}+\frac{2 \pi i}{m}} T_{n}(x) d x \geqslant \frac{2 m \sin \frac{n \beta}{2 m}}{n \beta}+\Xi-1
$$

and

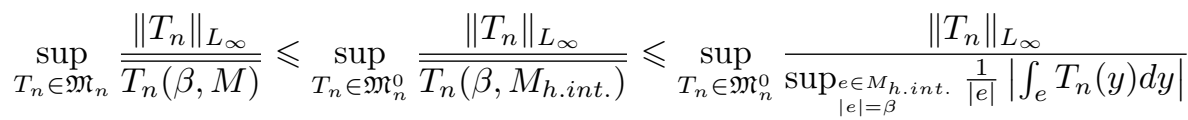

$$
\begin{aligned}
& \leqslant \sup _{1 \leqslant \Xi<\infty} \frac{\Xi}{\frac{2 m \sin \frac{n \beta}{2 m}}{n \beta}+\Xi-1}=\frac{n \beta}{2 m \sin \frac{n \beta}{2 m}},
\end{aligned}
$$

the required inequality.

Consider $T_{n}(x)=\cos n x$. For this function $m=n, \max \left|T_{n}(x)\right|=1$ and for $0<\beta<2 \pi$,

$$
\sup _{\substack{e \in M \\|e| \geqslant \beta}} \frac{1}{|e|}\left|\int_{e} T_{n}(y) d y\right|=\sup _{\substack{e \in M_{h . i n t .} \\|e| \geqslant \beta}} \frac{1}{|e|}\left|\int_{e} T_{n}(y) d y\right|=\frac{n}{\beta} \int_{-\frac{\beta}{2 n}}^{\frac{\beta}{2 n}} \cos n x d x=\frac{2 \sin \frac{\beta}{2}}{\beta},
$$

i.e., inequality (3.18) is sharp.

The proof of inequality (3.19) is similar to the proof above using

$$
\sup _{T_{n} \in \mathfrak{M}_{n}^{0}} \frac{\left\|T_{n}\right\|_{L_{\infty}}}{\overline{T_{n}\left(\beta, M_{\text {int. }}\right)}} \leqslant \sup _{T_{n} \in \mathfrak{M}_{n}^{0}} \frac{\left\|T_{n}\right\|_{L_{\infty}}}{\sup _{\substack{e \in M_{i n t .} \\|e|=\beta}} \frac{1}{|e|}\left|\int_{e} T_{n}(y) d y\right|} \leqslant \frac{n \beta}{2 \sin \frac{n \beta}{2}} .
$$

The next theorem plays an important role in approximation theory (see Stechkin [St] and Nikol'skii [Ni] in case $\beta=\pi / n)$. In particular, it generalizes Bernstein's inequality $\left\|T_{n}^{\prime}\right\|_{\infty} \leqslant n\left\|T_{n}\right\|_{\infty}$.

Corollary 3.10. Let $n \in \mathbb{N}$. We have

$$
\sup _{T_{n} \in \mathfrak{M}_{n}} \frac{\left\|T_{n}^{\prime}\right\|_{L_{\infty}}}{\left\|T_{n}(x+\beta)-T_{n}(x)\right\|_{L_{\infty}}}=\left(\frac{n}{2 \sin \frac{\beta n}{2}}\right), \quad 0<\beta<\frac{2 \pi}{n} .
$$

Proof. Without loss of generality, assume $\max _{x \in[-\pi, \pi)} T_{n}^{\prime}(x)=T_{n}^{\prime}(0) \geqslant 1$. The estimate from above follows from (3.20):

$$
\frac{\left\|T_{n}^{\prime}\right\|_{L_{\infty}}}{\max _{x \in[0,2 \pi)}\left|T_{n}(x+\beta)-T_{n}(x)\right|}=\frac{\left\|T_{n}^{\prime}\right\|_{L_{\infty}}}{\beta \max _{x \in[0,2 \pi)} \frac{1}{\beta}\left|\int_{x}^{x+\beta} T_{n}^{\prime}(y) d y\right|} \leqslant \frac{n}{2 \sin \frac{\beta n}{2}} .
$$

To prove the estimate from below, we consider $T_{n}(x)=\sin n x$.

\section{Sharp Nikol'skit and Bernstein-Nikol'skit inequalities in the Lorentz and Net SPACES}

We start this section with Nikol'skii's inequalities in the Lorentz spaces $L_{p, q} \equiv L_{p, q}(\mathbb{T})$.

Theorem 4.1. Let $0<p<\infty, 0<q \leqslant \infty$, and $n \in \mathbb{N}$. Let also $T_{n} \in \mathfrak{M}_{n}$ and $\frac{2 \pi}{m}$ be a minimal period of $T_{n}$. We have

$$
\begin{gathered}
\left\|T_{n}\right\|_{L_{\infty}} \leqslant\left(\frac{n}{m}\right)^{1 / p} \sup _{0 \leqslant \alpha \leqslant 1} \frac{1+\alpha}{\|\cos (\cdot)+\alpha\|_{L_{p, q}}}\left\|T_{n}\right\|_{L_{p, q}} \\
\left\|T_{n}^{(k)}\right\|_{L_{\infty}} \leqslant\left(\frac{n}{m}\right)^{1 / p} \frac{n^{k}}{\inf _{\alpha \in[0,1]}\|\cos n(\cdot)+\alpha\|_{L_{p, q}}}\left\|T_{n}\right\|_{L_{p, q}} .
\end{gathered}
$$


Inequalities (4.1) and (4.2) are sharp; they become equalities, respectively, for $T_{n, i}(x)=\cos n x+\alpha_{i}, \quad i=1,2$, where $\alpha_{1}$ is the point where the maximum of

$$
\frac{1+\alpha}{\|\cos (\cdot)+\alpha\|_{L_{p, q}}}, \quad \alpha \in[0,1]
$$

is attained and $\alpha_{2}$ is the point where the minimum of

$$
\|\cos (\cdot)+\alpha\|_{L_{p, q}}, \quad \alpha \in[0,1]
$$

is attained.

Remark 4.2. Note that in the papers [BKP1] and [BKP2] it is proved that

$$
\sup _{n \in \mathbb{N}} \sup _{T_{n} \in \mathfrak{M}_{n}} \frac{\left\|T_{n}\right\|_{L_{r}}}{n^{1 / p-1 / r}\left\|T_{n}\right\|_{L_{p}}}=\sup _{0 \leqslant \alpha \leqslant 1} \frac{\|\cos (\cdot)+\alpha\|_{L_{r}}}{\|\cos (\cdot)+\alpha\|_{L_{p}}}, \quad 0<p<r \leqslant \infty
$$

and

$$
\sup _{n \in \mathbb{N} T_{n} \in \operatorname{Mip}_{n}} \frac{\left\|T_{n}^{(k)}\right\|_{L_{\infty}}}{n^{k+1 / p}\left\|T_{n}\right\|_{L_{p}}}=\left(\|\cos (\cdot)\|_{L_{p}}\right)^{-1}, \quad 1 \leqslant p<\infty, k \in \mathbb{N} .
$$

Therefore, inequalities (4.1) and (4.2) supplement (4.3) and (4.4) for the case of the Lorentz spaces (take $p=q$ ). At the same time, considering minimal period of polynomials allows us to prove sharp Nikol'skii and Bernstein-Nikol'skii inequalities (4.1) and (4.2). The sharp constant in (4.1) was also discussed in [DP, Rem. 6.3].

We will need the following technical result.

Lemma 4.3. Suppose $0<p<\infty$ and $0<q \leqslant \infty$; then

$$
\sup _{0 \leqslant \alpha<\infty} \frac{1+\alpha}{\|\cos (\cdot)+\alpha\|_{L_{p, q}}}=\sup _{0 \leqslant \alpha \leqslant 1} \frac{1+\alpha}{\|\cos (\cdot)+\alpha\|_{L_{p, q}}}
$$

and

$$
\inf _{0 \leqslant \alpha<\infty}\|\cos n(\cdot)+\alpha\|_{L_{p, q}}=\inf _{0 \leqslant \alpha \leqslant 1}\|\cos n(\cdot)+\alpha\|_{L_{p, q}} .
$$

Proof. Let $\alpha \geqslant 1$. Let us find the extremal point of the function

$$
g(\alpha)=\frac{(1+\alpha)^{q}}{\|\cos (\cdot)+\alpha\|_{L_{p, q}}^{q}} .
$$

Since

$$
(\cos (\cdot)+\alpha)^{*}(t)=(\cos (\cdot)+1)^{*}(t)+\alpha-1=\cos \frac{t}{2}+\alpha,
$$

we get

$$
g^{\prime}(\alpha)=\left(q(1+\alpha)^{q-1} \int_{0}^{2 \pi} t^{\frac{q}{p}-1}\left(\cos \frac{t}{2}+\alpha\right)^{q-1}\left(\cos \frac{t}{2}-1\right) d t\right)\|\cos (\cdot)+\alpha\|_{L_{p, q}}^{-2 q}<0 .
$$

Hence, $g(\alpha) \leqslant g(1)$, and (4.5) follows. Inequality (4.6) can be shown similarly.

Proof of Theorem 4.1. Since $\frac{\left\|T_{n}\right\|_{L_{\infty}}}{\left\|T_{n}\right\|_{L_{p, q}}}$ is invariant under multiplication by $\gamma \in \mathbb{R} \backslash\{0\}$, we have

$$
\sup _{T_{n} \in \mathfrak{M}_{n}} \frac{m^{1 / p}\left\|T_{n}\right\|_{L_{\infty}}}{n^{1 / p}\left\|T_{n}\right\|_{L_{p, q}}}=\sup _{T_{n} \in \mathfrak{M}_{n}^{0}} \frac{m^{1 / p}\left\|T_{n}\right\|_{L_{\infty}}}{n^{1 / p}\left\|T_{n}\right\|_{L_{p, q}}}
$$

where $\mathfrak{M}_{n}^{0}$ is defined by (3.10).

For $T_{n} \in \mathfrak{M}_{n}^{0}$ we can assume that $\Xi:=\max T_{n}(x) \geqslant 1$. Let $\frac{2 \pi}{m}$ be the minimal period of $T_{n}$. By Lemma 2.3 ,

$$
T_{n}^{\star}(\beta) \geqslant(\cos n x+\Xi-1)^{\star}(\beta) \quad 0<\beta<2 \pi / n,
$$


and hence,

$$
\begin{aligned}
\left\|T_{n}\right\|_{L_{p, q}} & =m^{1 / p}\left(\int_{0}^{2 \pi / m} t^{\frac{q}{p}}\left(T^{*}(t m)\right)^{q} \frac{d t}{t}\right)^{1 / q} \\
& =m^{1 / p}\left(\int_{0}^{2 \pi / m} t^{\frac{q}{p}}\left(T^{\star}(t)\right)^{q} \frac{d t}{t}\right)^{1 / q} \\
& \geqslant m^{1 / p}\left(\int_{0}^{2 \pi / n} t^{\frac{q}{p}}\left(T^{\star}(t)\right)^{q} \frac{d t}{t}\right)^{1 / q} \\
& \geqslant m^{1 / p}\left(\int_{0}^{2 \pi / n} t^{\frac{q}{p}}\left((\cos n x+\Xi-1)^{\star}(t)\right)^{q} \frac{d t}{t}\right)^{1 / q} .
\end{aligned}
$$

Using Lemma 2.1(e), we have

$$
\left\|T_{n}\right\|_{L_{p, q}} \geqslant m^{1 / p} n^{-1 / p}\left(\int_{0}^{2 \pi} t^{\frac{q}{p}}\left((\cos x+\Xi-1)^{*}(t)\right)^{q} \frac{d t}{t}\right)^{1 / q} .
$$

Then

$$
\begin{aligned}
\sup _{\substack{T_{n} \in \mathfrak{M}_{n}^{0} \\
\Xi \geqslant 1}} \frac{m^{1 / p}\left\|T_{n}\right\|_{L_{\infty}}}{n^{1 / p}\left\|T_{n}\right\|_{L_{p, q}}} & \leqslant \sup _{\Xi \geqslant 1} \frac{\Xi}{\|\cos (\cdot)+\Xi-1\|_{L_{p, q}}} \\
& =\sup _{0 \leqslant \alpha<\infty} \frac{1+\alpha}{\|\cos (\cdot)+\alpha\|_{L_{p, q}}} .
\end{aligned}
$$

By Lemma 4.3, we obtain

$$
\sup _{T_{n} \in \mathfrak{M}_{n}} \frac{m^{1 / p}\left\|T_{n}\right\|_{L_{\infty}}}{n^{1 / p}\left\|T_{n}\right\|_{L_{p, q}}} \leqslant \sup _{0 \leqslant \alpha<\infty} \frac{1+\alpha}{\|\cos (\cdot)+\alpha\|_{L_{p, q}}}=\sup _{0 \leqslant \alpha \leqslant 1} \frac{1+\alpha}{\|\cos (\cdot)+\alpha\|_{L_{p, q}}} .
$$

To show the sharpness of (4.1), take $T_{n}(x)=\cos n x+\alpha_{1}$.

Let us now show the accuracy of (4.2). Estimating as above and using Bernstein's inequality, we get

$$
\begin{aligned}
\sup _{T_{n} \in \mathfrak{M}_{n}} \frac{m^{1 / p}\left\|T_{n}^{(k)}\right\|_{L_{\infty}}}{n^{k+1 / p}\left\|T_{n}\right\|_{L_{p, q}}} & =\sup _{T_{n} \in \mathfrak{M}_{n}^{0}} \frac{m^{1 / p}\left\|T_{n}^{(k)}\right\|_{L_{\infty}}}{n^{k+1 / p}\left\|T_{n}\right\|_{L_{p, q}}} \\
& \leqslant \sup _{T_{n} \in \mathfrak{M}_{n}^{0}} \frac{\left\|n^{-k}\left(T_{n}-(\Xi-1)\right)^{(k)}\right\|_{L_{\infty}}}{n^{1 / p}\left(\int_{0}^{2 \pi / n}\left(t^{\frac{1}{p}} T_{n}^{\star}(t)\right)^{q} \frac{d t}{t}\right)^{1 / q}} \\
& \leqslant \sup _{T_{n} \in \mathfrak{M}_{n}^{0}} \frac{1}{n^{1 / p}\left(\int_{0}^{2 \pi / n}\left(t^{\frac{1}{p}} T_{n}^{\star}(t)\right)^{q} \frac{d t}{t}\right)^{1 / q}} \\
& \leqslant \sup _{0 \leqslant \alpha<\infty} \frac{1}{\left(\int_{0}^{2 \pi}\left(t^{\frac{1}{p}}(\cos (\cdot)+\alpha)^{*}(t)\right)^{q} \frac{d t}{t}\right)^{1 / q}}=\sup _{0 \leqslant \alpha \leqslant 1} \frac{1}{\|\cos (\cdot)+\alpha\|_{L_{p, q}}}
\end{aligned}
$$

The polynomial $T_{n}(x)=\cos n x+\alpha_{2}$ provides the sharpness of (4.2).

The following remark gives the $\left(L_{p}, L_{p_{1}}\right)$-Nikol'skii inequality.

Remark 4.4. Let $0<p<p_{1}<\infty$ and $0<q_{1}, q \leqslant \infty$ such that $q / p=q_{1} / p_{1}$. Let $2 \pi / m$ be a minimal period of $T_{n} \in \mathfrak{M}_{n}$. Then

In particular,

$$
\left\|T_{n}\right\|_{L_{p_{1}, q_{1}}} \leqslant\left(\frac{n}{m}\right)^{1 / p-1 / p_{1}}\left(\sup _{0 \leqslant \alpha \leqslant 1} \frac{1+\alpha}{\|\cos (\cdot)+\alpha\|_{L_{p, q}}}\right)^{1-p / p_{1}}\left\|T_{n}\right\|_{L_{p, q}}
$$

$$
\left\|T_{n}\right\|_{L_{p_{1}}} \leqslant\left(\frac{n}{m}\right)^{1 / p-1 / p_{1}}\left(\sup _{0 \leqslant \alpha \leqslant 1} \frac{1+\alpha}{\|\cos (\cdot)+\alpha\|_{L_{p}}}\right)^{1-p / p_{1}}\left\|T_{n}\right\|_{L_{p}} .
$$


To finish this section, we give Nikol'skii's inequality for the net spaces.

Theorem 4.5. Let $0<p<\infty, 0<q \leqslant \infty$, and a net $M$ contain $M_{h . i .}$, the set of all harmonic intervals. Let $T_{n} \in \mathfrak{M}_{n}$ and $\frac{2 \pi}{m}$ be the minimal period of $T_{n}$. We have

$$
\begin{gathered}
\left\|T_{n}\right\|_{L_{\infty}} \leqslant\left(\frac{n}{m}\right)^{1 / p}\left(\left(\int_{0}^{2 \pi}\left(2 t^{\frac{1}{p}-1} \sin \frac{t}{2}\right)\right)^{q} \frac{d t}{t}\right)^{-1 / q}\left\|T_{n}\right\|_{N_{p, q}(M)}, \quad q<\infty ; \\
\left\|T_{n}\right\|_{L_{\infty}} \leqslant\left(\frac{n}{m}\right)^{1 / p}\left(\sup _{0<\alpha<\pi}(2 \alpha)^{\frac{1}{p}} \frac{\sin \alpha}{\alpha}\right)^{-1}\left\|T_{n}\right\|_{N_{p, \infty}(M) .}
\end{gathered}
$$

Inequalities (4.8) and (4.9) are sharp; they become equalities for $T_{n}(x)=\cos n x$.

Remark 4.6. An advantage of considering the net spaces is, in particular, that the constants in inequalities (4.8) and (4.9) do not have supremum form as in inequalities (4.1) and (4.2). On the other hand, if $M$ is the collection of all compact sets, then $N_{p, q}(M)=L_{p, q}, 1<p \leqslant \infty$; see [NT]. Moreover, $\left\|T_{n}\right\|_{N_{p, q}(M)} \leqslant$ $\left\|T_{n}^{* *}\right\|_{L_{p, q}}$ for any $p$ and $q$, where $T_{n}^{* *}(x)=\frac{1}{x} \int_{0}^{x} T_{n}^{*}(t) d t$.

Proof. Let $q<\infty$. Using (3.18), we get

$$
\begin{aligned}
\sup _{T_{n} \in \mathfrak{M}_{n}} \frac{m^{1 / p}\left\|T_{n}\right\|_{L_{\infty}}}{n^{1 / p}\left\|T_{n}\right\|_{N_{p, q}(M)}} & \leqslant \sup _{T_{n} \in \mathfrak{M}_{n}} \frac{m^{1 / p}\left\|T_{n}\right\|_{L_{\infty}}}{n^{1 / p}\left(\int_{0}^{2 \pi m / n}\left(t^{1 / p} \overline{T_{n}(t, M)}\right)^{q} \frac{d t}{t}\right)^{1 / q}} \\
& \leqslant \sup _{T_{n} \in \mathfrak{M}_{n}} \frac{m^{1 / p}}{n^{1 / p}\left(\int_{0}^{2 \pi m / n}\left(t^{1 / p} \frac{2 m}{n t} \sin \frac{n t}{2 m}\right)^{q} \frac{d t}{t}\right)^{1 / q}} \\
& =\left(\left(\int_{0}^{2 \pi}\left(2 t^{1 / p} \frac{\sin \frac{t}{2}}{t}\right)\right)^{q} \frac{d t}{t}\right)^{-1 / q} .
\end{aligned}
$$

If $q=\infty$, similarly, using again (3.18), we have

$$
\begin{aligned}
\sup _{T_{n} \in \mathfrak{M}_{n}} \frac{m^{1 / p}\left\|T_{n}\right\|_{L_{\infty}}}{n^{1 / p}\left\|T_{n}\right\|_{N_{p, \infty}(M)}} & \leqslant \sup _{T_{n} \in \mathfrak{M}_{n}} \frac{\left\|T_{n}\right\|_{L_{\infty}}}{\sup _{0<\beta<2 \pi m / n}\left(\left(\frac{n \beta}{m}\right)^{\frac{1}{p}} \overline{T_{n}(\beta, M)}\right)} \\
& \leqslant \sup _{T_{n} \in \mathfrak{M}_{n}} \frac{1}{\sup _{0<\beta<2 \pi m / n}\left(\left(\frac{n \beta}{m}\right)^{\frac{1}{p}} \frac{2 m}{n \beta} \sin \frac{n \beta}{2 m}\right)}=\left(\sup _{0<\alpha<\pi}(2 \alpha)^{\left.\frac{1}{p} \frac{\sin \alpha}{\alpha}\right)^{-1} .}\right.
\end{aligned}
$$

Considering $T_{n}(x)=\cos n x$ gives sharpness of inequalities (4.8) and (4.9).

\section{REMEZ InEQUALities in the MULtidimensional CASE}

Let $d \in \mathbb{N}$. The following multidimensional Remez inequality for trigonometric polynomials

$$
T_{n}(x)=\sum_{|\mathbf{k}| \leqslant n} c_{\mathbf{k}} e^{i(\mathbf{k}, x)}, \quad \mathbf{k}=\left(k_{1}, \cdots, k_{d}\right) \in \mathbb{Z}^{d}, \quad c_{\mathbf{k}} \in \mathbb{C}, \quad x \in \mathbb{T}^{d},
$$

where $|\mathbf{k}|=\|\mathbf{k}\|_{\ell_{\infty}}=\max \left\{\left|k_{1}\right|, \cdots,\left|k_{d}\right|\right\}$, is well known: For any Lebesgue measurable set $B \subset \mathbb{T}^{d}$ such that $|B|<(\pi / 2)^{d}$ we have

$$
\left\|T_{n}\right\|_{L_{\infty}\left(\mathbb{T}^{d}\right)} \leqslant \exp \left(4 d n|B|^{1 / d}\right)\left\|T_{n}\right\|_{L_{\infty}\left(\mathbb{T}^{d} \backslash B\right)} .
$$

For $d \geqslant 1$ the proof is given in [DP, $\mathrm{Kr}]$; the proof is by induction.

We consider more general trigonometric polynomials. Let $\mathbf{n}=\left(n_{1}, \cdots, n_{d}\right) \in \mathbb{Z}^{d}$ and

$$
\mathfrak{M}_{\mathbf{n}}(d)=\mathfrak{M}_{n_{1} \cdots n_{d}}(d)=\left\{T_{\mathbf{n}}: \mathbb{R} \ni T_{\mathbf{n}}(x)=\sum_{\left|k_{1}\right| \leqslant n_{1}} \cdots \sum_{\left|k_{d}\right| \leqslant n_{d}} c_{\mathbf{k}} e^{i(\mathbf{k}, x)}, \quad \mathbf{k} \in \mathbb{Z}^{d}, \quad c_{\mathbf{k}} \in \mathbb{C}, \quad x \in \mathbb{T}^{d}\right\}
$$


Note that Remark 3.1 holds for multivariate polynomials from $\mathfrak{M}_{\mathbf{n}}(d)$, that is, Remez's inequality can be studied in the form $T_{\mathbf{n}}^{*}(0) \leqslant C(\beta) T_{\mathbf{n}}^{*}(\beta), \beta>0$, where $f^{*}$ is the decreasing rearrangement of $f$ on $\mathbb{T}^{d}$, i.e., $f^{*}(t)=\inf \left\{\sigma: \mu\left\{x \in \mathbb{T}^{d}:|f(x)|>\sigma\right\} \leqslant t\right\}$ and $\mu$ is the $d$-dimensional Lebesgue measure.

Below we prove analogues of the one-dimensional Remez inequality. For the sake of simplicity, we consider only the case when $d=2$.

Theorem 5.1. Let $\mathbf{n}=\left(n_{1}, n_{2}\right) \in \mathbb{N}^{2}$ and $T_{\mathbf{n}}\left(x_{1}, x_{2}\right) \in \mathfrak{M}_{\mathbf{n}}(2)$. Then

$$
T_{\mathbf{n}}^{*}(0) \leqslant \frac{1}{\cos \sqrt{\frac{n_{1} n_{2} \beta}{2}}} T_{\mathbf{n}}^{*}(\beta), \quad \text { where } \quad 0 \leqslant \beta<\frac{\pi^{2}}{2 n_{1} n_{2}} .
$$

Proof. Let

Without loss of generality we assume that

$$
T_{\mathbf{n}}\left(x_{1}, x_{2}\right)=\sum_{k_{1}=-n_{1}}^{n_{1}} \sum_{k_{2}=-n_{2}}^{n_{2}} c_{k_{1}, k_{2}} \exp \left(i k_{1} x_{1}+i k_{2} x_{2}\right) .
$$

$$
\max T_{\mathbf{n}}\left(x_{1}, x_{2}\right)-\min T_{\mathbf{n}}\left(x_{1}, x_{2}\right)=2
$$

and

$$
T_{\mathbf{n}}(0,0)=\max T_{\mathbf{n}}\left(x_{1}, x_{2}\right):=\Xi \geqslant 1 .
$$

Let $\tau=\frac{p}{q} \in \mathbb{Q}, p \in \mathbb{Z}$, and $q \in \mathbb{N}$. Consider the polynomial $T_{\mathbf{n}}\left(x_{1}, x_{2}\right)$ on the line $x_{1}=x, x_{2}=\tau x$, i.e.,

$$
T_{\mathbf{n}}(x, \tau x)=\sum_{k_{1}=-n_{1}}^{n_{1}} \sum_{k_{2}=-n_{2}}^{n_{2}} c_{k_{1}, k_{2}} \exp \left(i\left(k_{1} q+k_{2} p\right) \frac{x}{q}\right)=: \widetilde{T}\left(\frac{x}{q}\right),
$$

where $\widetilde{T}(y)$ is the trigonometric polynomial of degree at most $n_{1} q+n_{2}|p|$ with respect to $y$. Using Lemma 2.2 ,

$$
\widetilde{T}(y) \geqslant \cos \left(n_{1} q+n_{2}|p|\right) y+\Xi-1, \quad-\frac{\pi}{n_{1} q+n_{2}|p|}<y<\frac{\pi}{n_{1} q+n_{2}|p|} .
$$

Let $z=\left(n_{1} q+n_{2}|p|\right) y$, i.e., $y=\frac{z}{\left(n_{1} q+n_{2}|p|\right)}$,

$$
\cos z+\Xi-1 \leqslant \widetilde{T}\left(\frac{z}{n_{1} q+n_{2}|p|}\right)=T_{\mathbf{n}}\left(\frac{q z}{n_{1} q+n_{2}|p|}, \frac{p z}{n_{1} q+n_{2}|p|}\right)
$$

where $-\pi<z<\pi$.

Denote

$$
Q_{z}=Q_{z}(\mathbf{n})=\left\{\left(x_{1}, x_{2}\right):\left|x_{1} n_{1}+x_{2} n_{2}\right| \leqslant z,\left|x_{1} n_{1}-x_{2} n_{2}\right| \leqslant z\right\}, \quad z>0 .
$$

Then inequality (5.3) implies

$$
T_{\mathbf{n}}\left(x_{1}, x_{2}\right) \geqslant \cos z+\Xi-1 \geqslant 0, \quad 0 \leqslant z \leqslant \frac{\pi}{2}
$$

for any $\left(x_{1}, x_{2}\right) \in\left\{\left(x_{1}, x_{2}\right): x_{2}=\tau x_{1},\left(x_{1}, x_{2}\right) \in \partial Q_{z}\right\}$ and $\tau \in \mathbb{Q}$. Here $\partial Q_{z}$ is the boundary of $Q_{z}$.

Since $T_{\mathbf{n}}$ is continuous,

$$
T_{\mathbf{n}}\left(x_{1}, x_{2}\right) \geqslant \cos z+\Xi-1 \geqslant 0, \quad 0 \leqslant z \leqslant \frac{\pi}{2}
$$

holds for any points of $Q_{z}$. Indeed, if now $\left(x_{1}^{0}, x_{2}^{0}\right) \in Q_{z}$, there exists $0 \leqslant z_{0} \leqslant z$ such that $\left(x_{1}^{0}, x_{2}^{0}\right) \in \partial Q_{z_{0}}$. Therefore, on the boundary $\partial Q_{z_{0}}$, we get

$$
T_{\mathbf{n}}\left(x_{1}^{0}, x_{2}^{0}\right) \geqslant \cos z_{0}+\Xi-1 \geqslant \cos z+\Xi-1 .
$$

Hence,

$$
T_{\mathbf{n}}\left(x_{1}, x_{2}\right) \geqslant \cos z+\Xi-1 \geqslant 0, \quad 0 \leqslant z \leqslant \frac{\pi}{2}
$$

for any $\left(x_{1}, x_{2}\right) \in Q_{z}$ and then

Finally,

$$
T_{\mathbf{n}}^{*}\left(\mu\left(Q_{z}\right)\right) \geqslant \cos z+\Xi-1 \geqslant 0 .
$$

$$
\sup _{T_{\mathbf{n}} \in \mathfrak{M}_{\mathbf{n}}(2)} \frac{T_{\mathbf{n}}^{*}(0)}{T_{\mathbf{n}}^{*}\left(\mu\left(Q_{z}\right)\right)} \leqslant \sup _{\Xi \geqslant 1} \frac{\Xi}{\cos z+\Xi-1}=\frac{1}{\cos z}
$$


and since $\mu\left(Q_{z}\right)=\frac{2 z^{2}}{n_{1} n_{2}}=\beta$, we arrive at (5.2).

In the general case $(d \geqslant 2)$ the proof is similar.

Remark 5.2. Let $d \geqslant 2, \mathbf{n} \in \mathbb{N}^{d}$, and $T_{\mathbf{n}} \in \mathfrak{M}_{\mathbf{n}}(d)$. Then

$$
T_{\mathbf{n}}^{*}(0) \leqslant \frac{1}{\cos \sqrt[d]{\beta \prod_{j=1}^{d} \frac{j n_{j}}{2}}} T_{\mathbf{n}}^{*}(\beta),
$$

where $0 \leqslant \beta<\frac{\pi^{d}}{\prod_{j=1}^{d} j n_{j}}$.

In particular, we have the following generalization of (5.1) for $T_{\mathbf{n}} \in \mathfrak{M}_{\mathbf{n}}(d)$ :

$$
\left\|T_{\mathbf{n}}\right\|_{L_{\infty}\left(\mathbb{T}^{d}\right)} \leqslant \exp \left(0,5 d\left(n_{1} \ldots n_{d}|B|\right)^{1 / d}\right)\left\|T_{\mathbf{n}}\right\|_{L_{\infty}\left(\mathbb{T}^{d} \backslash B\right)}, \quad \mu(B)=|B|<\prod_{j=1}^{d} \frac{2}{j n_{j}} .
$$

Indeed, since $\cos t \exp (t) \geqslant 1$ for $0<t \leqslant 1$, then for $d \geqslant 2$

$$
\frac{1}{\cos \sqrt[d]{|B| \prod_{j=1}^{d} \frac{j n_{j}}{2}}} \leqslant \exp \left(\sqrt[d]{|B| \prod_{j=1}^{d} \frac{j n_{j}}{2}}\right) \leqslant \exp \left(0.5 d\left(n_{1} \ldots n_{d}|B|\right)^{1 / d}\right), \quad|B|<\prod_{j=1}^{d} \frac{2}{j n_{j}} .
$$

Let us now present the multidimensional Nikol'skii inequality in the Lorentz spaces (cf. [DP]).

Corollary 5.3. Let $0<p<\infty, 0<q \leqslant \infty, \mathbf{n}=\left(n_{1}, \cdots, n_{d}\right) \in \mathbb{N}^{d}, d \geqslant 2$ and $T_{\mathbf{n}} \in \mathfrak{M}_{\mathbf{n}}(d)$. Then

$$
\sup _{T_{\mathbf{n}} \in \mathfrak{M}_{\mathbf{n}}} \frac{\left\|T_{\mathbf{n}}\right\|_{L_{\infty}\left(\mathbb{T}^{d}\right)}}{\left(\prod_{j=1}^{d} n_{j}\right)^{1 / p}\left\|T_{\mathbf{n}}\right\|_{L_{p, q}\left(\mathbb{T}^{d}\right)}} \leqslant\left(\frac{d !}{2^{d}}\right)^{1 / p}\left(d \int_{0}^{\frac{\pi}{2}}\left(t^{\frac{d}{p}} \cos t\right)^{q} \frac{d t}{t}\right)^{-\frac{1}{q}} .
$$

Proof. Making use of Theorem 5.1 and Remark 5.2 for $T_{\mathbf{n}} \in \mathfrak{M}_{\mathbf{n}}$, we get

$$
\begin{aligned}
\left\|T_{\mathbf{n}}\right\|_{L_{p, q}\left(\mathbb{T}^{d}\right)} & =\left(\int_{0}^{(2 \pi)^{d}}\left(t^{\frac{1}{p}} T_{\mathbf{n}}^{*}(t)\right)^{q} \frac{d t}{t}\right) \\
& \geqslant\left(\int_{0}^{\frac{\pi^{d}}{\Pi_{j=1}^{j} j n_{j}}}\left(t^{\frac{1}{p}} T_{\mathbf{n}}^{*}(t)\right)^{q} \frac{d t}{t}\right)^{\frac{1}{q}} \geqslant\left(\int_{0}^{\frac{\pi^{d}}{\Pi_{j=1}^{j n_{j}}}}\left(t^{\frac{1}{p}} \cos \sqrt[d]{t \prod_{j=1}^{d} \frac{j n_{j}}{2}}\right)^{q} \frac{d t}{t}\right)^{\frac{1}{q}} T_{\mathbf{n}}^{*}(0) \\
& =\left(\prod_{j=1}^{d} \frac{j n_{j}}{2}\right)^{-1 / p}\left(d \int_{0}^{\frac{\pi}{2}}\left(t^{\frac{d}{p}} \cos t\right)^{q} \frac{d t}{t}\right)^{\frac{1}{q}} T_{\mathbf{n}}^{*}(0),
\end{aligned}
$$

and we arrive at (5.6).

In some cases the integral on the right-hand side of (5.6) can be simply calculated. For example, for $d=2$, we have

$$
\begin{aligned}
\left\|T_{\mathbf{n}}\right\|_{\infty} & \leqslant \frac{n_{1} n_{2}}{4\left(\frac{\pi}{2}-1\right)}\left\|T_{\mathbf{n}}\right\|_{L_{1}}, \\
\left\|T_{\mathbf{n}}\right\|_{\infty} & \leqslant \frac{2 \sqrt{n_{1} n_{2}}}{\sqrt{\pi^{2}-4}}\left\|T_{\mathbf{n}}\right\|_{L_{2}}, \\
\left\|T_{\mathbf{n}}\right\|_{\infty} & \leqslant \frac{\sqrt{n_{1} n_{2}}}{2^{3 / 2}}\left\|T_{\mathbf{n}}\right\|_{L_{2,1}} .
\end{aligned}
$$

Similar to Remark 4.4 we can prove the following $\left(L_{p}, L_{p_{1}}\right)$-Nikol'skii inequality for $T_{\mathbf{n}} \in \mathfrak{M}_{\mathbf{n}}(d), d \geqslant 2$,

$$
\left\|T_{\mathbf{n}}\right\|_{L_{p_{1}}\left(\mathbb{T}^{d}\right)} \leqslant C^{1-p / p_{1}}\left(\prod_{j=1}^{d} n_{j}\right)^{\frac{1}{p}-\frac{1}{p_{1}}}\left\|T_{\mathbf{n}}\right\|_{L_{p}\left(\mathbb{T}^{d}\right)}, \quad 0<p<p_{1}<\infty
$$

where $C$ is the constant in the right-hand side of (5.6) with $p=q$. 
Finally, we note that Remez type inequalities hold not only for trigonometric polynomials but also for wider classes of functions. Denote by $\mathcal{E}_{\nu, \mu}, \nu, \mu>0$ the collection of functions $f \in L_{\infty}\left(\mathbb{R}^{2}\right)$ such that $\frac{\partial^{2} f}{\partial x_{1}^{\alpha_{1}} \partial x_{2}^{\alpha_{2}}} \in C\left(\mathbb{R}^{2}\right), \alpha_{1}+\alpha_{2}=2, \alpha_{i} \geqslant 0$ and

$$
\left\|\frac{\partial^{2} f}{\partial x_{1}^{\alpha_{1}} \partial x_{2}^{\alpha_{2}}}\right\|_{L_{\infty}\left(\mathbb{R}^{2}\right)} \leqslant \nu^{\alpha_{1}} \mu^{\alpha_{2}}\|f\|_{L_{\infty}\left(\mathbb{R}^{2}\right)}, \quad \alpha_{1}+\alpha_{2}=2, \alpha_{i} \geqslant 0 .
$$

This class in particular contains all functions of exponential type $(\nu, \mu)$ with regards to $x_{1}$ and $x_{2}$, respectively.

Theorem 5.4. Let $\nu, \mu>0$ and $0 \leqslant \beta<\frac{4}{\nu \mu}$. For any $f \in \mathcal{E}_{\nu, \mu}$ we have

$$
\sup _{f \in \mathcal{E}_{\nu, \mu}} \frac{f^{*}(0)}{f_{\nu, \mu}^{*}(\beta)} \leqslant\left(1-\frac{(\nu \mu \beta)}{4}\right)^{-1} .
$$

Proof. Without loss of generality we assume that

$$
\max _{(x, y) \in \mathbb{R}^{2}}|f(x, y)|=f(0,0)=1
$$

Then since $(0,0)$ is an extreme point, taking into account the definition of $\mathcal{E}_{\nu, \mu}$ and Taylor's formula, there exists $\left(c_{1}, c_{2}\right) \in(-|x|,|x|) \times(-|y|,|y|)$ such that

$$
f(x, y)=f(0,0)+\frac{1}{2}\left(\frac{\partial^{2} f}{\partial x^{2}}\left(c_{1}, c_{2}\right) x^{2}+2 \frac{\partial^{2} f}{\partial x \partial y}\left(c_{1}, c_{2}\right) x y+\frac{\partial^{2} f}{\partial y}\left(c_{1}, c_{2}\right) y^{2}\right) \geqslant 1-\frac{1}{2}(\nu|x|+\mu|y|)^{2} .
$$

Suppose $z=|x| \nu+|y| \mu$, then using the same argument as in the proof of Theorem 5.1,

$$
f(x, y) \geqslant 1-\frac{z^{2}}{2} \geqslant 0, \quad 0<z<\sqrt{2}
$$

for any point $(x, y)$ belonging to $Q_{z}=\{(x, y):|x| \nu+|y| \mu \leqslant z\}$. Finally, for $0 \leqslant \beta<\frac{4}{\nu \mu}$ we have

$$
f^{*}(\beta) \geqslant 1-\frac{\nu \mu \beta}{4}>0
$$

and therefore (5.7) follows.

\section{REFERENCES}

[An] V. Andrievskii, A note on a Remez-type inequality for trigonometric polynomials. J. Approx. Theory 116(2) (2002), $416-424$.

[BKP1] V. Babenko, V. Kofanov, S. Pichugov, Inequalities of Kolmogorov type and some their applications in approximation theory, Rendiconti del Circolo Matematico di Palermo Serie II, Suppl. 52 (1998), 223-237.

[BKP2] V. Babenko, V. Kofanov, S. Pichugov, Comparison of rearrangements and Kolmogorov-Nagy type inequalities for periodic functions, Approximation Theory: A volume dedicated to Bl. Sendov (B. Bojanov, Ed.), DARBA, Sofia (2002), $24-53$.

[BS] C. Bennett, R. Sharpley, Interpolation of Operators, Academic Press, 1988.

[Be] S. N. Bernstein, On one class of interpolation formulas (Russian), Izv. Ak. Nauk, 9 (1931), 1151-1161.

[BE] P. Borwein, T. Erdelyi, Polynomials and polynomial inequalities, Springer, New York, 1995.

[DP] Z. Ditzian, A. Prymak, Nikol'skii inequalities for Lorentz spaces, Rocky Mountain Journal of Mathematics, 40(1) (2010), 209-223.

[Er1] T. Erdélyi, Remez-type inequalities on the size of generalized polynomials, J. London Math. Soc. (2) 45 (1992), 255-264.

[Er2] T. Erdélyi, Remez-type inequalities and their applications, J. Comput. Appl. Math. 47 (1993), 167-210.

[Er3] T. Erdélyi, George Lorentz and inequalities in approximation, St. Petersbg. Math. J. 21(3) (2010), 365-405.

[EMN] T. Erdélyi, A. Máté, P. Nevai, Inequalities for generalized nonnegative polynomials, Constr. Approx. 8(2) (1992), 241-255.

[Ga] M. I. Ganzburg, Polynomial inequalities on measurable sets and their applications, Constr. Approx. 17 (2001), 275-306.

[Kr] A. Kroó, On Remez-type inequalities for polynomials in $R^{m}$ and $C^{m}$, Anal. Math. 27(1) (2001), 55-70.

[LGM] G.G. Lorentz, M. von Golitschek, Y. Makovoz, Constructive approximation: advanced problems. Springer, 1996.

[MT] G. Mastroianni, V. Totik, Weighted polynomial inequalities with doubling and $A_{\infty}$ weights, Constr. Approx. 16(1) (2000), 37-71.

[Na] F. L. Nazarov, Local estimates for exponential polynomials and their applications to inequalities of the uncertainty principle type, Algebra i Analiz 5(4) (1993), 3-66; translation in St. Petersburg Math. J. 5(4) (1994), 663-717.

[Ni] S. M. Nikol'skii, A generalization of one inequality by S. N. Bernstein (Russian), Dokl. Akad. Nauk SSSR, 60 (1948), $1507-1510$. 
[Nu] E.D. Nursultanov, Net spaces and inequalities of Hardy-Littlewood type, Sb. Math. 189, No.3 (1998), 399-419; translation from Mat. Sb. 189(3) (1998), 83-102.

[NA] E.D. Nursultanov, T.U. Aubakirov, Interpolation theorem for stochastic processes, Eurasian Math. J., 1(1) (2010), 8-16.

[NT] E. Nursultanov, S. Tikhonov, Net spaces and boundedness of integral operators, J. of Geometric Analysis, 21(4) (2011), 950-981.

[Re] E. Remes, Sur une propriété extremale des polynômes de Tchebychef (French), Commun. Inst. Sci. Math. et Mecan., Univ. Kharkoff et Soc. Math. Kharkoff, IV. Ser. 13 (1936), 93-95. Online: http://www.math.technion.ac.il/hat/fpapers/remezppr.pdf

[St] S. B. Stechkin, A generalization of some inequalities by S. N. Bernstein (Russian), Dokl. Akad. Nauk SSSR, 60 (1948), $1511-1514$.

[SU] S. B. Stechkin, P. L. Ulyanov, Sequences of convergence for series, Trudy Mat. Inst. Steklov, 86 (1965), 3-83. English translation in Proc. Steklov Inst. Math., (1967), 1-85.

E. Nursultanov

L.N. Gumilyov Eurasian National University and

Kazakh Branch of Moscow State University

MunatPasova, 7

010010 Astana KaZaKhStan

E-mail address: er-nurs@yandex.ru

S. TikHONOV

iCREA and Centre de Recerca Matemàtica

Facultat de Cincies, UAB

08193 Bellaterra

BARCELONA, Spain

E-mail address: stikhonov@crm.cat 